\title{
Path subcoalgebras, finiteness properties and quantum groups
}

\author{
Sorin Dăscălescu, Miodrag C. Iovanov, and Constantin Năstăsescu
}

\begin{abstract}
We study subcoalgebras of path coalgebras that are spanned by paths (called path subcoalgebras) and subcoalgebras of incidence coalgebras, and propose a unifying approach for these classes. We discuss the left quasi-co-Frobenius and the left co-Frobenius properties for these coalgebras. We classify the left co-Frobenius path subcoalgebras, showing that they are direct sums of certain path subcoalgebras arising from the infinite line quiver or from cyclic quivers. We investigate which of the co-Frobenius path subcoalgebras can be endowed with Hopf algebra structures, in order to produce some quantum groups with non-zero integrals, and we classify all these structures over a field with primitive roots of unity of any order. These turn out to be liftings of quantum lines over certain not necessarily abelian groups.
\end{abstract}

Mathematics Subject Classification (2010). 16T15, 16T05, 05C38, 06A11, $16 \mathrm{~T} 30$.

Keywords. Incidence coalgebra, path coalgebra, co-Frobenius coalgebra, quasi-co-Frobenius coalgebra, balanced bilinear form, quantum group, integral.

\section{Introduction and Preliminaries}

Let $K$ be an arbitrary field. A quadratic algebra is a quotient of a free noncommutative algebra $K\left\langle x_{1}, \ldots, x_{n}\right\rangle$ in $n$ variables by an ideal $I$ generated by elements of degree 2 . The usual commutative polynomial ring is such an example, with $I$ generated by $x_{i} x_{j}-x_{j} x_{i}$. Quadratic algebras are important in many places in mathematics, and one relevant class of such objects consists of Koszul algebras and Koszul duals of quadratic algebras. More generally, one can consider quotients $K\left\langle x_{1}, \ldots, x_{n}\right\rangle / I$ for ideals $I$ generated by homogeneous elements. Several algebras occur in this way in topology, noncommutative geometry, representation theory, or theoretical physics (see the examples and references in [7]). Such are the cubic Artin-Schreier regular algebras $\mathbb{C}\langle x, y\rangle /\left(a y^{2} x+b y x y+a x y^{2}+c x^{3}, a x^{2} y+b x y x+a y x^{2}+x y^{3}\right)$ in noncommutative projective algebraic geometry (see [3]), the skew-symmetrizer killing algebras $\mathbb{C}\left\langle x_{1}, \ldots, x_{n}\right\rangle /\left(\sum_{\sigma \in \Sigma_{p}} \operatorname{sgn}(\sigma) x_{i_{\sigma(1)}} \ldots x_{i_{\sigma(p)}}\right)$ (the ideal we factor out has $\left(\begin{array}{l}n \\ p\end{array}\right)$ generators, each one corresponding to some fixed $1 \leq i_{1}<\cdots<i_{p} \leq$ $n$ ) for a fixed $2 \leq p \leq n$, in representation theory (see [6]), or the Yang-Mills algebras $\mathbb{C}\left\langle\nabla_{0}, \ldots, \nabla_{n}\right\rangle /\left(\sum_{\lambda, \mu} g^{(\lambda, \mu)}\left[\nabla_{\lambda}\left[\nabla_{\mu}, \nabla_{\nu}\right]\right]\right)$ (with $\left(g^{(\lambda, \mu)}\right)_{\lambda, \mu}$ an invertible symmetric real matrix, and the ideal we factor out has $n+1$ generators, as $0 \leq v \leq n$ ) 
in theoretical physics (see [14]), to name a few. More generally, one could start with a quiver $\Gamma$, and define path algebras with relations by taking quotients of the path algebra $K[\Gamma]$ by an ideal (usually) generated by homogeneous elements, which are obtained as linear combinations of paths of the same length. Note that the examples above are of this type: the free algebra with $n$ elements can be thought as the path algebra of the quiver $\Gamma$ with one vertex 1 (which becomes the unit in the algebra) and $n$ arrows $x_{1}, \ldots, x_{n}$ starting and ending at 1 ; the relations are then given by linear combinations of paths of the same length. This approach, for example, allows the generalization of $N$-Koszulity to quiver algebras with relations, see [17].

We aim to study a general situation which is dual to the ones above, but is also directly connected to it. If $\Gamma$ is a quiver, the path algebra $K[\Gamma]$ of $\Gamma$ plays an important role in the representation theory of $\Gamma$. The underlying vector space of the path algebra also has a coalgebra structure, which we denote by $K \Gamma$ and call the path coalgebra of $\Gamma$. One motivation for replacing path algebras by path coalgebras is the following: given an algebra $A$, and its category of finite dimensional representations, one is often led to considering the category $\operatorname{Ind}(A)$ generated by all these finite dimensional representations (direct limits of finite dimensional representations). $\operatorname{Ind}(A)$ is well understood as the category of comodules over the finite dual coalgebra $A^{0}$ of $A$ (also called the algebra of representative functions on $A$ ), and it cannot be regarded as a full category of modules over a ring unless $A$ is finite dimensional. Such situations extend beyond the realm of pure algebra, encompassing representations of compact groups, affine algebraic groups or group schemes, differential affine groups, Lie algebras and Lie groups, infinite tensor categories, etc.

Another reason why the study of path coalgebras is interesting is that any pointed coalgebra embeds into the path coalgebra of the associated Gabriel quiver, see [24], [12]. On the other hand, if $X$ is a locally finite partially ordered set, the incidence coalgebra $K X$ provides a good framework for interpreting several combinatorial problems in terms of coalgebras, as explained by Joni and Rota in [22]. There are several features common to path coalgebras and incidence coalgebras. They are both pointed, the group-like elements recover the vertices of the quiver, respectively the points of the ordered set, the injective envelopes of the simple comodules have similar descriptions, etc. Moreover, as we show later in Section 5, Proposition 5.1, any incidence coalgebra embeds in a path coalgebra, and in many situations it has a basis where each element is a sum of paths of the same length. We note that this is precisely the dual situation to that considered above: for algebras one considers a path algebra with homogeneous relations, that is, $K[\Gamma]$ factored out by an ideal generated by homogeneous elements, i.e., sums of paths of the same length with coefficients. For a coalgebra one considers subcoalgebras of the path coalgebra of $\Gamma$ such that the coalgebra has a basis consisting of linear combinations of paths of the same length ("homogeneous" elements; more generally, a coalgebra generated by such elements).

In this paper we study Frobenius type properties for path coalgebras, incidence coalgebras and certain subcoalgebras of them. Recall that a coalgebra $C$ is called left co-Frobenius if $C$ embeds in $C^{*}$ as a left $C^{*}$-module. Also, $C$ is called left 
quasi-co-Frobenius if $C$ embeds in a free module as a left $C^{*}$-module. The (quasi)co-Frobenius properties are interesting for at least three reasons. Firstly, coalgebras with such properties have rich representation theories. Secondly, for a Hopf algebra $H$, it is true that $H$ is left quasi-co-Frobenius if and only if $H$ is left co-Frobenius, and this is also equivalent to $H$ having non-zero left (or right) integrals. Co-Frobenius Hopf algebras are important since they generalize the algebra of representative functions $R(G)$ on a compact group $G$, which is a Hopf algebra whose integral is the left Haar integral of $G$. Moreover, more recent generalizations of these have been made to compact and locally compact quantum groups (whose representation categories are not necessarily semisimple). Thus co-Frobenius coalgebras may be the underlying coalgebras for interesting quantum groups with non-zero integrals. Thirdly, by keeping in mind the duality with Frobenius algebras in the finite dimensional case, co-Frobenius coalgebras have connections to topological quantum field theory.

We propose an approach leading to similar results for path coalgebras and incidence coalgebras, and which also points out the similarities between these as mentioned above. It will follow from our results that a path coalgebra (or an incidence coalgebra) is left (quasi)-co-Frobenius if and only if the quiver consists only of isolated points, i.e., the quiver does not have arrows (respectively the order relation is the equality). Thus the left co-Frobenius coalgebras arising from path coalgebras or incidence coalgebras are just group-like coalgebras. In order to discover more interesting left co-Frobenius coalgebras, we focus our attention to classes of coalgebras larger than just path coalgebras and incidence coalgebras. On one hand we consider subcoalgebras of path coalgebras which have a linear basis consisting of paths. We call these path subcoalgebras. On the other hand, we look at subcoalgebras of incidence coalgebras; any such coalgebra has a basis consisting of segments. In Section 2 we apply a classical approach to the (quasi)-co-Frobenius property. It is known that a coalgebra $C$ is left co-Frobenius if and only if there exists a left non-degenerate $C^{*}$-balanced bilinear form on $C$. Also, $C$ is left quasi-co-Frobenius if and only if there exists a family $\left(\beta_{i}\right)_{i \in I}$ of $C^{*}$-balanced bilinear forms on $C$ such that for any non-zero $x \in C$ there is $i \in I$ with $\beta_{i}(x, C) \neq 0$. We describe the balanced bilinear forms on path subcoalgebras and subcoalgebras of incidence coalgebras. Such a description was given in [16] for the full incidence coalgebra, and in [5] for certain matrix-like coalgebras. In Section 3 we use this description and an approach using the injective envelopes of the simple comodules to show that a coalgebra lying in one of the two classes is left quasi-co-Frobenius if and only if it is left co-Frobenius, and to give several equivalent conditions including combinatorial ones (just in terms of paths of the quiver, or segments of the ordered set).

In Section 4 we classify all possible left co-Frobenius path subcoalgebras. We construct some classes of left co-Frobenius coalgebras $K\left[\mathbb{A}_{\infty}, r\right]$ and $K\left[\mathbb{A}_{0, \infty}, r\right]$ starting from the infinite line quiver $\mathbb{A}_{\infty}$, and a class of left co-Frobenius coalgebras $K\left[\mathbb{C}_{n}, s\right]$ starting from cyclic quiver $\mathbb{C}_{n}$. Our result says that any left co-Frobenius path subcoalgebra is isomorphic to a direct sum of coalgebras of types $K\left[\mathbb{A}_{\infty}, r\right]$, $K\left[\mathbb{A}_{0, \infty}, r\right], K\left[\mathbb{C}_{n}, s\right]$ or $K$, with special quivers $\mathbb{A}_{\infty}, \mathbb{A}_{0, \infty}, \mathbb{C}_{n}$ and $r, s$ being certain 
general types of functions on these quivers. For subcoalgebras of incidence coalgebras we do not have a complete classification in the left co-Frobenius case. We show in Section 5 that more complicated examples than the ones in the path subcoalgebra case can occur for subcoalgebras of incidence coalgebras, and a much larger class of such coalgebras is to be expected. Also, we give several examples of co-Frobenius subcoalgebras of path coalgebras, which are not path subcoalgebras, and moreover, examples of pointed co-Frobenius coalgebras which are not isomorphic to any one of the above mentioned classes. In Section 6 we discuss the possibility of defining Hopf algebra structures on the path subcoalgebras that are left and right co-Frobenius, classified in Section 4. The main reason for asking this question is the interest in constructing quantum groups with non-zero integrals, whose underlying coalgebras are path subcoalgebras. We answer completely this question in the case where $K$ contains primitive roots of unity of any positive order. Thus we determine all possible co-Frobenius path subcoalgebras admitting a Hopf algebra structure. Moreover, we describe up to an isomorphism all such Hopf algebra structures. It turns out that they are liftings of quantum lines over certain not necessarily abelian groups. In particular, this also answers the question of finding the Hopf algebra structures on finite dimensional path subcoalgebras and on quotients of finite dimensional path algebras by ideals spanned by paths. Our results contain, as particular cases, some results of [10], where finite quivers $\Gamma$ and finite dimensional path subcoalgebras $C$ of $K \Gamma$ are considered, such that $C$ contains all vertices and arrows of $\Gamma$. The coFrobenius coalgebras of this type are determined, and all Hopf algebra structures on them are described in [10]. These results follow from our more general Theorem 4.6 and Theorem 6.4. We note that Hopf algebra structures on incidence coalgebras have been of great interest for combinatorics, see for example [25], [1]. We also note that the classification of path coalgebras that admit a graded Hopf algebra structure was done in [13], see also [18] for a different point of view on Hopf algebra structures on path algebras. In particular, some of the examples in the classification have deep connections with homological algebra: the monoidal category of chain $s$-complexes of vector spaces over $K$ is monoidal equivalent to the category of comodules of $K\left[\mathbb{A}_{\infty} \mid s\right]$, a subclass of the Hopf algebras classified here ([21], [8]).

We also note that the unifying approach we propose here seems to suggest that in general for pointed coalgebras interesting methods and results could be obtained provided one can find some suitable bases with properties resembling those of paths in quiver algebras or segments in incidence coalgebras.

Throughout the paper $\Gamma=\left(\Gamma_{0}, \Gamma_{1}\right)$ will be a quiver. $\Gamma_{0}$ is the set of vertices, and $\Gamma_{1}$ is the set of arrows of $\Gamma$. If $a$ is an arrow from the vertex $u$ to the vertex $v$, we denote $s(a)=u$ and $t(a)=v$. A path in $\Gamma$ is a finite sequence of arrows $p=a_{1} a_{2} \ldots a_{n}$, where $n \geq 1$, such that $t\left(a_{i}\right)=s\left(a_{i+1}\right)$ for any $1 \leq i \leq n-1$. We will write $s(p)=s\left(a_{1}\right)$ and $t(p)=t\left(a_{n}\right)$. Also the length of such a $p$ is length $(p)=n$. Vertices $v$ in $\Gamma_{0}$ are also considered as paths of length zero, and we write $s(v)=t(v)=v$. If $q$ and $p$ are two paths such that $t(q)=s(p)$, we consider the path $q p$ by taking the arrows of $q$ followed by the arrows of $p$. We 
denote by $K \Gamma$ the path coalgebra, which is the vector space with a basis consisting of all paths in $\Gamma$, and comultiplication $\Delta$ defined by $\Delta(p)=\sum_{q r=p} q \otimes r$ for any path $p$, and counit $\epsilon$ defined by $\epsilon(v)=1$ for any vertex $v$, and $\epsilon(p)=0$ for any path of positive length. In particular, the arrows $x$ between two vertices $v$ and $w$, i.e., $s(x)=v, t(x)=w$, are the nontrivial elements of $P_{w, v}$, the space of $(w, v)$ skew-primitive elements: $\Delta(x)=v \otimes x+x \otimes w$. When we use Sweedler's sigma notation $\Delta(p)=\sum p_{1} \otimes p_{2}$ for a path $p$, we always take representations of the sum such that all $p_{1}$ 's and $p_{2}$ 's are paths.

We also consider partially ordered sets $(X, \leq)$ which are locally finite, i.e., the interval $[x, y]=\{z \mid x \leq z \leq y\}$ is finite for any $x \leq y$. The incidence $K$-coalgebra of $X$, denoted by $K X$, is the $K$-vector space with basis $\left\{e_{x, y} \mid x, y \in X, x \leq y\right\}$, and comultiplication $\Delta$ and counit $\epsilon$ defined by

$$
\Delta\left(e_{x, y}\right)=\sum_{x \leq z \leq y} e_{x, z} \otimes e_{z, y}, \quad \epsilon\left(e_{x, y}\right)=\delta_{x, y},
$$

for any $x, y \in X$ with $x \leq y$, where by $\delta_{x, y}$ we denote Kronecker's delta. The elements $e_{x, y}$ are called segments. Again, when we use Sweedler's sigma notation $\Delta(p)=\sum p_{1} \otimes p_{2}$ for a segment $p$, we always take representations of the sum such that all $p_{1}$ 's and $p_{2}$ 's are segments. Recall that the length of a segment $e_{x, y}$ is the maximum length $n$ of a chain $x=z_{0}<z_{1}<\cdots<z_{n}=y$.

For basic terminology and notation about coalgebras and Hopf algebras we refer to [15] and [23].

\section{Balanced bilinear forms for path subcoalgebras and for subcoalgebras of incidence coalgebras}

In the rest of the paper we will be interested in two classes of coalgebras more general than path coalgebras and incidence coalgebras. Thus we will study:

- Subcoalgebras of the path coalgebra $K \Gamma$ having a basis $\mathcal{B}$ consisting of paths in $\Gamma$. Such a coalgebra will be called a path subcoalgebra. Note that if $p \in \mathscr{B}$, then any subpath of $p$, in particular any vertex involved in $p$, lies in $\mathcal{B}$.

- Subcoalgebras of the incidence coalgebra $K X$. By [16], Proposition 1.1, any such subcoalgebra has a basis $\mathscr{B}$ consisting of segments $e_{x, y}$, and moreover, if $e_{x, y} \in \mathscr{B}$ and $x \leq a \leq b \leq y$, then $e_{a, b} \in \mathscr{B}$.

It is clear that for a coalgebra $C$ of one of these two types, the distinguished basis $\mathscr{B}$ consists of all paths (or segments) which are elements of $C$. Let $C$ be a coalgebra of one of these two types, with basis $\mathcal{B}$ as above. When we use Sweedler's sigma notation $\Delta(p)=\sum p_{1} \otimes p_{2}$ for $p \in \mathscr{B}$, we always consider representations of the sum such that all $p_{1}$ 's and $p_{2}$ 's are in $\mathcal{B}$.

A bilinear form $\beta: C \times C \rightarrow K$ is $C^{*}$-balanced if

$$
\sum \beta\left(p_{2}, q\right) p_{1}=\sum \beta\left(p, q_{1}\right) q_{2} \text { for any } p, q \in \mathcal{B} .
$$


It is clear that (1) is equivalent to the fact that for any $p, q \in \mathscr{B}$, the following three conditions hold:

$$
\begin{aligned}
& \beta\left(p_{2}, q\right)=\beta\left(p, q_{1}\right) \text { for those of the } p_{2} \text { 's and the } q_{1} \text { 's such that } p_{1}=q_{2}, \\
& \beta\left(p_{2}, q\right)=0 \text { for those } p_{2} \text { 's for which } p_{1} \text { is not equal to any } q_{2}, \\
& \beta\left(p, q_{1}\right)=0 \text { for those } q_{1} \text { 's for which } q_{2} \text { is not equal to any } p_{1} .
\end{aligned}
$$

In the following two sections we discuss separately path subcoalgebras and subcoalgebras of incidence coalgebras.

2.1. Path subcoalgebras. In this section we consider the case where $C$ is a path subcoalgebra. We note that if $\Gamma$ is acyclic, then for any paths $p$ and $q$ there is at most a pair $\left(p_{1}, q_{2}\right)$ (in (1)) such that $p_{1}=q_{2}$.

Denote by $\mathcal{F}$ the set of all paths $d$ satisfying the following three properties:

- $d=q p$ for some $q, p \in \mathscr{B}$.

- For any representation $d=q p$ with $q, p \in \mathscr{B}$, and any arrow $a \in \Gamma_{1}$, if $a p \in \mathscr{B}$ then $q$ must end with $a$.

- For any representation $d=q p$ with $q, p \in \mathscr{B}$, and any arrow $b \in \Gamma_{1}$, if $q b \in \mathscr{B}$ then $p$ starts with $b$.

Now we are able to describe all balanced bilinear forms on $C$.

Theorem 2.1. A bilinear form $\beta: C \times C \rightarrow K$ is $C^{*}$-balanced if and only if there is a family of scalars $\left(\alpha_{d}\right)_{d \in \mathcal{F}}$ such that, for any $p, q \in \mathscr{B}$,

$$
\beta(p, q)= \begin{cases}\alpha_{d} & \text { if } s(p)=t(q) \text { and } q p=d \in \mathscr{F}, \\ 0 & \text { otherwise. }\end{cases}
$$

In particular the set of all $C^{*}$-balanced bilinear forms on $C$ is in bijective correspondence to $K^{\mathcal{F}}$.

Proof. Assume that $\beta$ is $C^{*}$-balanced. If $p, q \in \mathscr{B}$ and $t(q) \neq s(p)$, then $\beta(p, q) s(p)$ appears in the left-hand side of (1), but $s(p)$ does not show up in the right-hand side, so $\beta(p, q)=0$. Let $\mathcal{P}$ be the set of all paths in $\Gamma$ for which there are $p, q \in \mathscr{B}$ such that $d=q p$. Let $d \in \mathcal{P}$ and let $d=q p=q^{\prime} p^{\prime}, p, q, p^{\prime}, q^{\prime} \in \mathscr{B}$ be two different decompositions of $d$, and say that, for example, length $\left(p^{\prime}\right)<\operatorname{length}(p)$. Then there is a path $r$ such that $p=r p^{\prime}$ and $q^{\prime}=q r$, and clearly $r \in \mathscr{B}$ since it is a subpath of $q^{\prime} \in B$. Use (2) for $p$ and $q^{\prime}$, for which there is an equality $p_{1}=q_{2}^{\prime}=r$ (and the corresponding $p_{2}=p^{\prime}$ and $\left.q_{1}^{\prime}=q\right)$, and find that $\beta\left(p^{\prime}, q^{\prime}\right)=\beta(p, q)$. Therefore, for any $d \in \mathcal{P}$ (not necessarily in $\mathscr{B}$ ) and any $p, q \in \mathscr{B}$ such that $d=q p$, the scalar $\beta(p, q)$ depends only on $d$. This shows that there is a family of scalars $\left(\alpha_{d}\right)_{d \in \mathcal{P}}$ such that $\beta(p, q)=\alpha_{d}$ for any $p, q \in \mathscr{B}$ with $q p=d$. 
Let $d \in \mathcal{P}$ such that $d=q p$ for some $p, q \in \mathscr{B}$, and there is an arrow $a \in \Gamma_{1}$ with $a p \in \mathscr{B}$, but $q$ does not end with $a$. That is, $q$ is not of the form $q=r a$ for some path $r \in \mathscr{B}$. We use (3) for the paths $a p \in \mathscr{B}$ and $q \in \mathscr{B}$, more precisely, for the term $(a p)_{1}=a$, which cannot be equal to any of the $q_{2}$ 's (otherwise $q$ would end with $a$ ), and we see that $\beta(p, q)=0$, i.e., $\alpha_{d}=0$.

Similarly, if $d \in \mathcal{P}, d=q p$ with $p, q \in \mathscr{B}$ and there is $b \in \Gamma_{1}$ with $q b \in B$ and $p$ not of the form $b r$ for some path $r$ (i.e., $p$ does not start with $b$ ), then we use (4) for $p$ and $q b$, and $(q b)_{2}=b$, and we find that $\beta(p, q)=0$, i.e., $\alpha_{d}=0$. In conclusion, $\alpha_{d}$ may be non-zero only for $d \in \mathscr{F}$.

Conversely, assume that $\beta$ is of the form indicated in the statement. We show that (2), (3) and (4) are satisfied. Let $p, q \in \mathscr{B}$ be such that $p_{1}=q_{2}=r$ for some $p_{1}$ and $q_{2}$ (from the comultiplication $\sum p_{1} \otimes p_{2}$ of $p$ and, respectively, the comultiplication $\sum q_{1} \otimes q_{2}$ of $\left.q\right)$. Then $p=r p^{\prime}$ and $q=q^{\prime} r$ for some $p^{\prime}, q^{\prime} \in \mathscr{B}$. Let $d=q^{\prime} r p^{\prime}$. If $d \in \mathscr{F}$, then $\beta\left(p^{\prime}, q\right)=\beta\left(p, q^{\prime}\right)=\alpha_{d}$, while if $d \notin \mathscr{F}$ we have $\beta\left(p^{\prime}, q\right)=\beta\left(p, q^{\prime}\right)=0$ by definition. Thus (2) holds. Now let $p, q \in \mathscr{B}$ and fix some $p_{2}$ (from the comultiplication $\sum p_{1} \otimes p_{2}$ of $p$ ) such that the corresponding $p_{1}$ is not equal to any $q_{2}$. If $s\left(p_{2}\right) \neq t(q)$, then clearly $\beta\left(p_{2}, q_{1}\right)=0$ by the definition of $\beta$. If $s\left(p_{2}\right)=t(q)$, then $d=q p_{2} \notin \mathscr{F}$. Indeed, let $r$ be a maximal path such that $p_{1}=e r$ for some path $e$ and $q$ ends with $r$, say $q=q^{\prime} r$. Note that $e$ has length at least 1 , since $p_{1}$ is not equal to any of the $q_{2}$ 's. Then the terminal arrow of $e$ cannot be the terminal arrow of $q^{\prime}$, and this shows that $d=p_{2} q=\left(p_{2} r\right) q^{\prime} \notin \mathcal{F}$. Then $\beta\left(p_{2}, q\right)=0$ and (3) is satisfied. Similarly, (4) is satisfied.

2.2. Subcoalgebras of incidence coalgebras. In this section we assume that $C$ is a subcoalgebra of the incidence coalgebra $K X$. Let $\mathscr{D}$ be the set of all pairs $(x, y)$ of elements in $X$ such that $x \leq y$ and there exists $x^{\prime}$ with $x \leq x^{\prime} \leq y$ and $e_{x, x^{\prime}}, e_{x^{\prime}, y} \in \mathscr{B}$. Fix $(x, y) \in \mathscr{D}$. Let

$$
U_{x, y}=\left\{u \mid x \leq u \leq y \text { and } e_{x, u}, e_{u, y} \in \mathscr{B}\right\}
$$

and define the relation $\sim$ on $U_{x, y}$ by $u \sim v$ if and only if there exist a positive integer $n$, and $u_{0}=u, u_{1}, \ldots, u_{n}=v$ and $z_{1}, \ldots, z_{n}$ in $U_{x, y}$, such that $z_{i} \leq u_{i-1}$ and $z_{i} \leq u_{i}$ for any $1 \leq i \leq n$. It is easy to see that $\sim$ is an equivalence relation on $U_{x, y}$. Let $U_{x, y} / \sim$ be the associated set of equivalence classes, and denote by $\left(U_{x, y} / \sim\right)_{0}$ the set of all equivalence classes $\mathcal{C}$ satisfying the following two conditions:

- If $u \in \mathcal{C}, v \in X$ satisfies $v \leq u$ and $e_{v, y} \in \mathscr{B}$, then $x \leq v$.

- If $u \in \mathscr{C}, v \in X$ satisfies $u \leq v$ and $e_{x, v} \in \mathscr{B}$, then $v \leq y$.

Now we can describe the balanced bilinear forms on $C$.

Theorem 2.2. A bilinear form $\beta: C \times C \rightarrow K$ is $C^{*}$-balanced if and only if there 
is a family of scalars $(\alpha €) \in \in \sqcup_{(x, y) \in \mathcal{D}}\left(U_{x, y} / \text { sim }\right)_{0}$ such that, for any $e_{t, y}, e_{x, z} \in \mathscr{B}$,

$$
\beta\left(e_{t, y}, e_{x, z}\right)=\left\{\begin{array}{l}
\alpha e \quad \text { if }(x, y) \in \mathcal{D}, z=t \in U_{x, y} \text { and the class } C \\
\quad \text { of } z \text { in } U_{x, y} / \sim \text { is in }\left(U_{x, y} / \sim\right)_{0}, \\
\text { otherwise. }
\end{array}\right.
$$

In particular the set of all $C^{*}$-balanced bilinear forms on $C$ is in bijective correspondence to $K_{(x, y) \in \mathscr{D}}\left(U_{x, y} / \sim\right)_{0}$.

Proof. Assume that $\beta$ is $C^{*}$-balanced. Fix some $x \leq y$ such that $U_{x, y} \neq \emptyset$. We first note that if $x \leq z \leq t \leq y$ and $z, t \in U_{x, y}$, then by applying (2) for $p=e_{z, y}$, $q=e_{x, t}$ and $p_{1}=q_{2}=e_{z, t}$, we find that $\beta\left(e_{t, y}, e_{x, t}\right)=\beta\left(e_{z, y}, e_{x, z}\right)$. Now let $u, v \in U_{x, y}$ such that $u \sim v$. Let $u_{0}=u, u_{1}, \ldots, u_{n}=v$ and $z_{1}, \ldots, z_{n}$ in $U_{x, y}$ such that $z_{i} \leq u_{i-1}$ and $z_{i} \leq u_{i}$ for any $1 \leq i \leq n$. By the above $\beta\left(e_{u_{i-1}, y}, e_{x, u_{i-1}}\right)=\beta\left(e_{u_{i}, y}, e_{x, u_{i}}\right)=\beta\left(e_{z_{i}, y}, e_{x, z_{i}}\right)$ for any $i$, and this implies that $\beta\left(e_{u, y}, e_{x, u}\right)=\beta\left(e_{v, y}, e_{x, v}\right)$. This shows that $\beta\left(e_{u, y}, e_{x, u}\right)$ takes the same value for any $u$ in the same equivalence class in $U_{x, y} / \sim$.

Now assume that for some $u \in U_{x, y}$ there is $v \in X$ such that $v \leq u, x \not \leq$ and $e_{v, y} \in \mathscr{B}$. Use (3) for $p=e_{v, y}, q=e_{x, u}$ and $p_{1}=e_{v, u}$. Note that $p_{1} \neq q_{2}$ for any $q_{2}$. We get $\beta\left(e_{u, y}, e_{x, u}\right)=0$.

Similarly, if $u \in U_{x, y}$, and there is $v \in X$ such that $u \leq v, v \not y$ and $e_{x, v} \in \mathcal{B}$, then using (4) for $p=e_{u, y}, q=e_{x, v}$ and $q_{2}=e_{u, v}$, we find that $\beta\left(e_{u, y}, e_{x, u}\right)=0$. We have thus showed that $\beta$ has the desired form.

Conversely, assume that $\beta$ has the indicated form. We show that it satisfies (2), (3) and (4). Let $p, q \in \mathcal{B}$ such that $p_{1}=q_{2}$ for some $p_{1}$ and $q_{2}$. Then $p=e_{z, y}, q=e_{x, t}$ and $p_{1}=q_{2}=e_{z, t}$ for some $x \leq z \leq t \leq y$. Clearly $t \sim z$, and let $\mathcal{C}$ be the equivalence class of $t$ in $U_{x, y} / \sim$. Then $\beta\left(p_{2}, q\right)=\beta\left(e_{t, y}, e_{x, t}\right)$ and $\beta\left(p, q_{1}\right)=\beta\left(e_{z, y}, e_{x, z}\right)$, and they are both equal to $\alpha \mathcal{C}$ if $\mathcal{C} \in\left(U_{x, y} / \sim\right)_{0}$, and to 0 if $\mathcal{(} \notin\left(U_{x, y} / \sim\right)_{0}$. Thus (2) is satisfied.

Let now $p=e_{z, y}, p_{1}=e_{z, t}, p_{2}=e_{t, y}$ and $q=e_{x, u}$ such that $p_{1} \neq q_{2}$ for any $q_{2}$. Then $\beta\left(p_{2}, q\right)=\beta\left(e_{t, y}, e_{x, u}\right)$. If $u \neq t$, this is clearly 0 . Let $u=t$. Then $x \notin z$, otherwise $p_{1}=q_{2}$ for some $q_{2}$. We have $t \in U_{x, y}$, but the equivalence class of $t$ in $U_{x, y} / \sim$ is not in $\left(U_{x, y} / \sim\right)_{0}$, since $e_{z, y} \in \mathscr{B}, z \leq t$, but $x \not z$. It follows that $\beta\left(p_{2}, q\right)=0$, and (3) holds. Similarly we can show that (4) holds.

\section{Left quasi-co-Frobenius path subcoalgebras and subcoalgebras of incidence coalgebras}

In this section we investigate when a path subcoalgebra of a path coalgebra or a subcoalgebra of an incidence coalgebra is left co-Frobenius. We keep the notation of Section 2. Thus $C$ will be either a path subcoalgebra of a path coalgebra $K \Gamma$, or a subcoalgebra of an incidence coalgebra $K X$. The distinguished basis of $C$ consisting 
of paths or segments will be denoted by $\mathcal{B}$. We note that in each of the two cases $\mathscr{B} \cap C_{n}$ is a basis of $C_{n}$, where $C_{0} \subseteq C_{1} \subseteq \cdots$ is the coradical filtration of $C$. The injective envelopes of the simple left (right) comodules were described in [26], Lemma 5.1, for incidence coalgebras and in [11], Corollary 6.3, for path coalgebras. It is easy to see that these descriptions extend to the following.

Proposition 3.1. (i) If $C$ is a path subcoalgebra, then for each vertex $v$ of $\Gamma$ such that $v \in C$, the injective envelope of the left (right) $C$-comodule $K v$ is (the $K$-span) $E_{l}(K v)=\langle p \in \mathscr{B} \mid t(p)=v\rangle$ (and $E_{r}(K v)=\langle p \in \mathcal{B} \mid s(p)=v\rangle$ respectively).

(ii) If $C$ is a subcoalgebra of the incidence coalgebra $K X$, then for any $a \in X$ such that $e_{a, a} \in C$ the injective envelope of the left (right) $C$-comodule $K e_{a, a}$ is (the $K$-span) $E_{l}\left(K e_{a, a}\right)=\left\langle e_{x, a} \mid x \in X, e_{x, a} \in C\right\rangle\left(\right.$ and $E_{r}\left(K e_{a, a}\right)=\left\langle e_{a, x}\right| x \in$ $\left.\left.X, e_{a, x} \in C\right\rangle\right)$.

The following shows that we have a good left-right duality for comodules generated by elements of the basis $\mathcal{B}$.

Lemma 3.2. (i) Let $C$ be a subcoalgebra of the incidence coalgebra $K X$, and let $e_{a, b} \in C$. Then $\left(C^{*} e_{a, b}\right)^{*} \cong e_{a, b} C^{*}$ as right $C^{*}$-modules (or left $C$-comodules).

(ii) Let $C$ be a path subcoalgebra of $K \Gamma$, and let $p$ be a path in $C$. Then $\left(C^{*} p\right)^{*} \cong p C^{*}$ as right $C^{*}$-modules (or left $C$-comodules).

Proof. (i) Clearly the set of all segments $e_{a, x}$ with $a \leq x \leq b$ is a basis of $C^{*} e_{a, b}$. Denote by $e_{a, x}^{*}$ the corresponding elements of the dual basis of $\left(C^{*} e_{a, b}\right)^{*}$. Since for $c^{*} \in C^{*}$ and $a \leq x, y \leq b$ we have

$$
\left(e_{a, x}^{*} c^{*}\right)\left(e_{a, y}\right)=\sum_{a \leq z \leq y} c^{*}\left(e_{z, y}\right) e_{a, x}^{*}\left(e_{a, z}\right)= \begin{cases}0 & \text { if } x \notin y, \\ c^{*}\left(e_{x, y}\right) & \text { if } x \leq y,\end{cases}
$$

we get

$$
e_{a, x}^{*} c^{*}=\sum_{x \leq y \leq b} c^{*}\left(e_{x, y}\right) e_{a, y}^{*} .
$$

On the other hand $e_{a, b} C^{*}$ has a basis consisting of all segments $e_{x, b}$ with $a \leq x \leq b$, and

$$
e_{x, b} c^{*}=\sum_{x \leq y \leq b} c^{*}\left(e_{x, y}\right) e_{y, b}
$$

Equations (5) and (6) show that the linear map $\phi:\left(C^{*} e_{a, b}\right)^{*} \rightarrow e_{a, b} C^{*}$ defined by $\phi\left(e_{a, x}^{*}\right)=e_{x, b}$, is an isomorphism of right $C^{*}$-modules.

(ii) Let $p=a_{1} \ldots a_{n}$ and $v=s(p)$. Denote $p_{i}=a_{1} \ldots a_{i}$ for any $1 \leq i \leq n$, and $p_{0}=v$. Then $\left\{p_{0}, p_{1}, \ldots, p_{n}\right\}$ is a basis of $C^{*} p$, and let $\left(p_{i}^{*}\right)_{0 \leq i \leq n}$ be the dual basis of $\left(C^{*} p\right)^{*}$. For any $0 \leq t \leq j \leq n$ denote by $\overline{p_{t, j}}$ the path such that $p_{j}=p_{t} \overline{p_{t, j}}$. Then a simple computation shows that $p_{i}^{*} c^{*}=\sum_{i \leq j \leq n} c^{*}\left(\overline{p_{i, j}}\right) p_{j}^{*}$ for any $i$ and any $c^{*} \in C^{*}$. 
On the other hand, $\left\{\overline{p_{i, n}} \mid 0 \leq i \leq n\right\}$ is a basis of $p C^{*}$, and it is easy to see that $\overline{p_{i, n}} c^{*}=\sum_{i \leq r \leq n} c^{*}\left(\overline{p_{i, r}}\right) \overline{p_{r, n}}$ for any $i$ and any $c^{*} \in C^{*}$. Then the linear map $\phi:\left(C^{*} p\right)^{*} \rightarrow p \bar{C}^{*}$ defined by $\phi\left(p_{i}^{*}\right)=\overline{p_{i, n}}$ for any $0 \leq i \leq n$ is an isomorphism of right $C^{*}$-modules.

For a path subcoalgebra $C$ let us denote by $R(C)$ the set of vertices $v$ in $C$ such that the set $\{p \in C \mid p$ path and $s(p)=v\}$ is finite (i.e., $E_{r}(K v)$ is finite dimensional) and contains a unique maximal path. Note that $v \in R(C)$ if and only if $E_{r}(K v)$ is finite dimensional and local. Indeed, if $E_{r}(K v)$ is finite dimensional and contains a unique maximal path $p=a_{1} \ldots a_{n}$, then keeping the notation from the proof of Lemma 3.2, we have that $E_{r}(K v)=C^{*} p$ and $C^{*} p_{n-1}=<p_{0}, \ldots, p_{n-1}>$ is the unique maximal $C^{*}$-submodule of $C^{*} p$. Conversely, if $E_{r}(K v)$ is finite dimensional and local with the unique maximal subcomodule $N$, then the set $\left(\mathscr{B} \cap E_{r}(K v)\right) / N$ is nonempty. If $p$ is a path which belongs to this set, $E_{r}(K v)=C^{*} p$. Then clearly $p$ is the unique maximal path in $\{q \in C \mid q$ path and $s(q)=v\}$.

Similarly, denote by $L(C)$ the set of vertices $v$ of $C$ such that $E_{l}(K v)$ is a finite dimensional local left $C$-comodule. Also, for each vertex $v \in R(C)$ let $r(v)$ denote the endpoint of the maximal path starting at $v$, and for $v \in L(C)$ let $l(v)$ be the starting point of the maximal path ending at $v$.

Similarly, for a subcoalgebra $C$ of the incidence coalgebra $K X$, let $R(C)$ be the set of all $a \in X$ for which $e_{a, a} \in C$ and the set $\left\{x \in X \mid a \leq x, e_{a, x} \in C\right\}$ is finite and has a unique maximal element, and $L(C)$ be the set of all $a \in X$ for which $e_{a, a} \in C$ and the set $\left\{x \in X \mid x \leq a, e_{x, a} \in C\right\}$ is finite and has a unique minimal element. As before, $R(C)$ (respectively $L(C)$ ) consists of those $a \in X$ for which $E_{r}\left(K e_{a, a}\right)$ (respectively, $E_{l}\left(K e_{a, a}\right)$ ) are local, hence generated by a segment. Here $r(a)=r\left(e_{a, a}\right)$ for $a \in R(C)$ denotes the maximum element in the set $\left\{x \mid x \geq a, e_{a, x} \in C\right\}$ and $l(a)$ for $a \in L(C)$ means the minimum of $\left\{x \mid x \leq a, e_{x, a} \in C\right\}$.

Proposition 3.3. (I) Let $C$ be a path subcoalgebra of the path coalgebra $K \Gamma$. Then the following are equivalent.

(a) $C$ is left co-Frobenius.

(b) C is left quasi-co-Frobenius.

(c) $R(C)$ consists of all vertices belonging to $C, r(R(C)) \subseteq L(C)$ and $\operatorname{lr}(v)=v$ for any vertex $v$ in $C$.

(d) For any path $q \in \mathscr{B}$ there exists a path $p \in \mathscr{B}$ such that $q p \in \mathcal{F}$ ( for $\mathcal{F}$ defined in the previous section).

(II) Let $C$ be a subcoalgebra of the incidence coalgebra $K X$. Then the following are equivalent.

(a) $C$ is left co-Frobenius.

(b) C is left quasi-co-Frobenius. 
(c) $R(C)$ consists of all $a \in X$ such that $e_{a, a} \in C, r(R(C)) \subseteq L(C)$ and $\operatorname{lr}(a)=a$ for all $a \in X$ with $e_{a, a} \in C$.

(d) For any segment $e_{x, z} \in C$ there exists $y \geq z$ such that $e_{z, y} \in C$ and the class of $z$ in $U_{x, y} / \sim$ lies in $\left(U_{x, y} / \sim\right)_{0}$.

Proof. (I) (a) $\Longrightarrow$ (b) is clear.

(b) $\Longrightarrow$ (c). We apply the QcF characterization of [20] and [21]. If $C$ is left QcF then for any vertex $v \in C$, there is a vertex $u \in C$ such that $E_{r}(K v) \cong E_{l}(K u)^{*}$. Hence $E_{r}(K v)$ is finite dimensional and local (by [19], Lemma 1.4), so $v \in R(C)$ and $E_{r}(K v)=C^{*} p$ for a path $p$ by the discussion preceding this Proposition. Let $t(p)=$ $w$. Then it is easy to see that the linear map $\phi: C^{*} p \rightarrow K w$ taking $p$ to $w$, and any other $q$ to 0 , is a surjective morphism of left $C^{*}$-modules. Since $E_{r}(K v) \cong E_{l}(K u)^{*}$, there is a surjective morphism of left $C^{*}$-modules $E_{l}(K u)^{*} \rightarrow K w$, inducing an injective morphism of right $C^{*}$-modules $(K w)^{*} \rightarrow E_{l}(K u)$. Since $(K w)^{*} \cong K w$ as right $C^{*}$-modules, and the socle of the comodule $E_{l}(K u)$ is $K u$, we must have $w=u$, and thus $u=r(v)$. By Lemma 3.2, $E_{l}(K u) \cong E_{r}(K v)^{*}=\left(C^{*} p\right)^{*} \cong p C^{*}$, so $E_{l}(K u)$ is generated by $p$, and this shows that $p$ is the unique maximal path ending at $u$. Hence, $u=r(v) \in L(X)$, and $l(u)=v$. Thus $l(r(v))=v$.

(c) $\Longrightarrow(\mathrm{d})$. Let $q \in \mathcal{B}$, and let $v=s(q)$. Since $v \in R(C)$, there exists a unique maximal path $d$ starting at $v$, and $d=q p$ for some path $p$. We show that $d \in \mathscr{F}$. Denote $t(d)=v^{\prime}$, and let $d=q^{\prime} p^{\prime}$ for some paths $q^{\prime}, p^{\prime}$ in $\mathscr{B}$. Let $u=t\left(q^{\prime}\right)=s\left(p^{\prime}\right)$. If there is an arrow $b$ (in $\Gamma_{1}$ ) starting at $u$, such that $q^{\prime} b \in \mathscr{B}$, then $q^{\prime} b$ is a subpath of $d$, since $d$ is the unique maximal path starting at $v$. It follows that $p^{\prime}$ starts with $b$. On the other hand, $v^{\prime}=r(v) \in L(C)$ and $l\left(v^{\prime}\right)=\operatorname{lr}(v)=v$, so $d$ is the unique maximal path in $\mathscr{B}$ ending at $v^{\prime}$. This shows that if an arrow $a$ (in $\Gamma_{1}$ ) ends at $u$, and $a p^{\prime} \in \mathscr{B}$, then $a p^{\prime}$ is a subpath of $d$, so the last arrow of $q^{\prime}$ is $a$. We conclude that $d \in \mathscr{F}$.

(d) $\Longrightarrow$ (a). Choose a family $\left(\alpha_{d}\right)_{d \in \mathscr{F}}$ of scalars, such that $\alpha_{d} \neq 0$ for any $d$. Associate a $C^{*}$-balanced bilinear form $B$ on $C$ to this family of scalars as in Theorem 2.1. Then $B$ is right non-degenerate, so $C$ is left co-Frobenius.

(II) (a) $\Longrightarrow$ (b) is clear; (b) $\Longrightarrow$ (c) is proved as the similar implication in (I), with paths replaced by segments.

(c) $\Longrightarrow$ (d). Let $e_{x, z} \in C$. If $r(x)=y$, then clearly $z \leq y$ and $e_{x, y} \in \mathscr{B}$, so $U_{x, y}=[x, y]$. Then any two elements in $U_{x, y}$ are equivalent with respect to $\sim$ (since they are both $\geq x$ ), so there is precisely one equivalence class in $U_{x, y} / \sim$, the whole of $U_{x, y}$. We show that this class lies in $\left(U_{x, y} / \sim\right)_{0}$. Indeed, if $u \in U_{x, y}, v \in X$, $v \leq u$ and $e_{v, y} \in \mathscr{B}$, then $v \in\left\{a \mid e_{a, y} \in \mathscr{B}\right\}$, and since $l(y)=l(r(x))=x$, we must have $x \leq v$. Also, if $u \in U_{x, y}, v \in X, u \leq v$ and $e_{x, v} \in \mathscr{B}$, then $v \in\left\{a \mid e_{x, a} \in \mathscr{B}\right\}$. Then $v \leq y$ since $r(x)=y$.

(d) $\Longrightarrow$ (a) follows as the similar implication in $(I)$ if we take into account Theorem 2.2. 
As a consequence we obtain the following result, which was proved for incidence coalgebras in [16].

Corollary 3.4. If $C=K \Gamma$, a path coalgebra, or $C=K X$, an incidence coalgebra, the following are equivalent.

(i) $C$ is co-semisimple (i.e., $\Gamma$ has no arrows for $C=K \Gamma$, and the order relation on $X$ is the equality for $C=K X$ ).

(ii) $C$ is left $Q c F$.

(iii) $C$ is left co-Frobenius.

(iv) $C$ is right $Q c F$.

(v) $C$ is right co-Frobenius.

As an immediate consequence we describe the situations where a finite dimensional path algebra is Frobenius. We note that the path algebra of a quiver $\Gamma$ (as well as the path coalgebra $K \Gamma$ ) has finite dimension if and only if $\Gamma$ has finitely many vertices and arrows, and there are no cycles.

Corollary 3.5. A finite dimensional path algebra is Frobenius if and only if the quiver has no arrows.

Proof. This follows from the fact that the dual of a finite dimensional path algebra is a path coalgebra, and by Corollary 3.4.

\section{Classification of left co-Frobenius path subcoalgebras}

Proposition 3.3 gives information about the structure of left co-Frobenius path subcoalgebras. The aim of this section is to classify these coalgebras. We first use Proposition 3.3 to give some examples of left co-Frobenius path subcoalgebras. These examples will be the building blocks for the classification.

Example 4.1. Let $\Gamma=\mathbb{A}_{\infty}$ be the quiver such that $\Gamma_{0}=\mathbb{Z}$ and there is precisely one arrow from $i$ to $i+1$ for any $i \in \mathbb{Z}$.

$$
\mathbb{A}_{\infty}: \cdots \longrightarrow \circ^{-1} \longrightarrow \circ^{0} \longrightarrow \circ^{1} \longrightarrow \circ^{2} \longrightarrow \cdots .
$$

For any $k<l$, let $p_{k, l}$ be the (unique) path from the vertex $k$ to the vertex $l$. Also denote by $p_{k, k}$ the vertex $k$. Let $r: \mathbb{Z} \rightarrow \mathbb{Z}$ be a strictly increasing function such that $r(n)>n$ for any $n \in \mathbb{Z}$. We consider the path subcoalgebra $K\left[\mathbb{A}_{\infty}, r\right]$ of $K \mathbb{A}_{\infty}$ with the basis

$$
\begin{aligned}
\mathscr{B} & =\bigcup_{n \in \mathbb{Z}}\left\{p \mid p \text { is a path in } \mathbb{A}_{\infty}, s(p)=n \text { and length }(p) \leq r(n)-n\right\} \\
& =\left\{p_{k, l} \mid k, l \in \mathbb{Z} \text { and } k \leq l \leq r(k)\right\} .
\end{aligned}
$$


Note that $K\left[\mathbb{A}_{\infty}, r\right]$ is indeed a subcoalgebra since

$$
\Delta\left(p_{k, l}\right)=\sum_{i=k}^{l} p_{k, i} \otimes p_{i, l}, \quad k \leq l .
$$

The counit is given by

$$
\varepsilon\left(p_{k, l}\right)=\delta_{k, l} \text {. }
$$

Note that this can also be seen as a subcoalgebra of the incidence coalgebra of $(\mathbb{N}, \leq)$, consisting of the segments $e_{k, l}$ for $k \leq l \leq r(k)$.

The construction immediately shows that the maximal path starting from $n$ is $p_{n, r(n)}$. Note that for each $n \in \mathbb{Z}, p_{n, r(n)}$ is the unique maximal path into $r(n)$. If there would be another longer path $p_{l, r(n)}$ into $r(n)$ in $K\left[\mathbb{A}_{\infty}, r\right]$, then $l<n$. Then, since $p_{l, r(n)}$ is among the paths in $K\left[\mathbb{A}_{\infty}, r\right]$ which start at $l$ we must have that it is a subpath of $p_{l, r(l)}$, and so $r(l) \geq r(n)$. But since $l<n$, this contradicts the assumption that $r$ is strictly increasing. Therefore, we see that the conditions of Proposition 3.3 are satisfied: $p_{n, r(n)}$ is the unique maximal path in the (finite) set of all paths starting from a vertex $n$, and it is simultaneously the unique maximal path in the (finite) set of all paths ending at $r(n)$. Therefore if $l: L(C)=\operatorname{Im}(r) \rightarrow R(C)$ is the function used in Proposition 3.3 for $C=K\left[\mathbb{A}_{\infty}, r\right]$ satisfies $l(r(n))=n$. This means that $K\left[\mathbb{A}_{\infty}, r\right]$ is a left co-Frobenius coalgebra.

$K\left[\mathbb{A}_{\infty}, r\right]$ is also right co-Frobenius if and only if there is a positive integer $s$ such that $r(n)=n+s$ for any $n \in \mathbb{Z}$. Indeed, if $r$ is of such a form, then $K\left[\mathbb{A}_{\infty}, r\right]$ is right co-Frobenius by the right-hand version of Proposition 3.3. Conversely, assume that $K\left[\mathbb{A}_{\infty}, r\right]$ is right co-Frobenius. If $r$ would not be surjective, let $m \in \mathbb{Z}$ which is not in the image of $r$. Then there is $n \in \mathbb{Z}$ such that $r(n)<m<r(n+1)$. The maximal path ending at $m$ is $p_{n+1, m}$. Indeed, this maximal path cannot start before $n$ (since then $p_{n, r(n)}$ would be a subpath of $p_{n, m}$ different from $\left.p_{n, m}\right)$, and $p_{n+1, m}$ is a path in $K\left[\mathbb{A}_{\infty}, r\right]$, as a subpath of $p_{n+1, r(n+1)}$. Hence $r(l(m))=r(n+1) \neq m$, and then $K\left[\mathbb{A}_{\infty}, r\right]$ could not be right co-Frobenius by the right-hand version of Proposition 3.3, a contradiction. Thus $r$ must be surjective, and then it must be of the form $r(n)=n+s$ for any $n$, where $s$ is an integer. Since $n<r(n)$ for any $n$, we must have $s>0$. For simplicity we will denote $K\left[\mathbb{A}_{\infty}, r\right]$ by $K\left[\mathbb{A}_{\infty} \mid s\right]$ in the case where $r(n)=n+s$ for any $n \in \mathbb{Z}$.

Example 4.2. Let $\Gamma=\mathbb{A}_{0, \infty}$ be the subquiver of $\mathbb{A}_{\infty}$ obtained by deleting all the negative vertices and the arrows involving them. Thus $\Gamma_{0}=\mathbb{N}$, the natural numbers (including 0).

$$
\mathbb{A}_{0, \infty}: \circ^{0} \longrightarrow \circ^{1} \longrightarrow \circ^{2} \longrightarrow \circ^{3} \longrightarrow \cdots \text {. }
$$

We keep the same notation for $p_{k, l}$ for $0 \leq k \leq l$. Let $r: \mathbb{N} \rightarrow \mathbb{N}$ be a strictly increasing function with $r(0)>0$ (so then $r(n)>n$ for any $n \in \mathbb{N}$ ), and define $K\left[\mathbb{A}_{0, \infty}, r\right]$ to be the path subcoalgebra of $K \mathbb{A}_{0, \infty}$ with basis $\left\{p_{k, l} \mid k, l \in \mathbb{N}, k \leq\right.$ $l \leq r(k)\}$. With the same arguments as in Example 4.1 we see that $K\left[\mathbb{A}_{0, \infty}, r\right]$ is a 
left co-Frobenius coalgebra. We note that $l(0)=0$, and then $r(l(0))=r(0)>0$. By a right-hand version of Proposition 3.3, this shows that $K\left[\mathbb{A}_{0, \infty}, r\right]$ is never right co-Frobenius.

Example 4.3. For any $n \geq 2$ we consider the quiver $\mathbb{C}_{n}$, whose vertices are the elements of $\mathbb{Z}_{n}=\{\overline{0}, \ldots, \overline{n-1}\}$, the integers modulo $n$, and there is one arrow from $\bar{i}$ to $\overline{i+1}$ for each $i$.

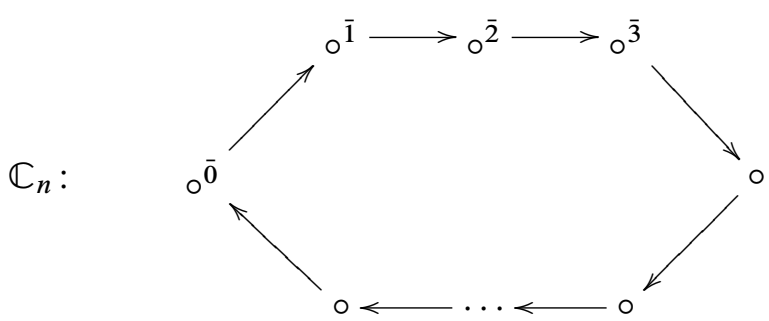

We also denote by $\mathbb{C}_{1}$ the quiver with one vertex, denoted by $\overline{0}$, and one arrow $\circ$, and by $\mathbb{C}_{0}$ the quiver with one vertex and no arrows.

Let $n \geq 1$ and $s>0$ be integers. Let $K\left[\mathbb{C}_{n}, s\right]$ be the path subcoalgebra of the path coalgebra $K \mathbb{C}_{n}$, spanned by all paths of length at most $s$. Denote by $q_{\bar{k} \mid l}$ the path (in $\mathbb{C}_{n}$ ) of length $l$ starting at $\bar{k}$, for any $\bar{k} \in \mathbb{Z}_{n}$ and $0<l \leq s$. Also denote by $q_{\bar{k} \mid 0}$ the vertex $\bar{k}$. Since the comultiplication and counit of $K \mathbb{C}_{n}$ are given by

$$
\Delta\left(q_{\bar{k} \mid l}\right)=\sum_{i=0}^{l} q_{\bar{k} \mid i} \otimes q_{\overline{k+i} \mid l-i}, \quad \varepsilon\left(q_{\bar{k} \mid l}\right)=\delta_{0, l},
$$

we see that indeed $K\left[\mathbb{C}_{n}, s\right]=<q_{\bar{k} \mid l} \mid \bar{k} \in \mathbb{Z}, 0 \leq l \leq s>$ is a subcoalgebra of $K \mathbb{C}_{n}$. Clearly $q_{\bar{k} \mid s}$ is the unique maximal path in $K\left[\mathbb{C}_{n}, s\right]$ starting at $\bar{k}$, so $\bar{k} \in R\left(K\left[\mathbb{C}_{n}, s\right]\right)$ and $r(\bar{k})=\overline{k+s}$. Also $\overline{k+s} \in L\left(K\left[\mathbb{C}_{n}, s\right]\right)$ and the maximal path ending at $\overline{k+s}$ is also $q_{\bar{k} \mid s}$, thus $\operatorname{lr}(\bar{k})=\bar{k}$, and by Proposition 3.3 we get that $K\left[\mathbb{C}_{n}, s\right]$ is a left co-Frobenius coalgebra. Since it has finite dimension $n(s+1)$, it is right co-Frobenius, too. This example was also considered in [10], 1.6.

For a path subcoalgebra $C \subseteq K \Gamma$, denote by $C \cap \Gamma$ the subgraph of $\Gamma$ consisting of arrows and vertices of $\Gamma$ belonging to $C$.

Lemma 4.4. If $C \subseteq K \Gamma$ is a left co-Frobenius path subcoalgebra, then $C \cap \Gamma=$ $\bigsqcup_{i} \Gamma_{i}$, a disjoint union of subquivers of $\Gamma$, where each $\Gamma_{i}$ is of one of types $\mathbb{A}_{\infty}$, $\mathbb{A}_{0, \infty}$ or $\mathbb{C}_{n}, n \geq 0$, and $C=\bigoplus_{i} C_{i}$, where $C_{i}$, a path subcoalgebra of $K \Gamma_{i}$, is the subcoalgebra of $C$ spanned by the paths of $\mathcal{B}$ contained in $\Gamma_{i}$.

Proof. Let $v$ be a vertex in $C \cap \Gamma$. By Proposition 3.3 there is a unique maximal path $p \in \mathscr{B}$ starting at $v$, and any path in $\mathscr{B}$ starting at $v$ is a subpath of $p$. This 
shows that at most one arrow in $\mathcal{B}$ starts at $v$ (the first arrow of $p$, if $p$ has length $>0$ ). We show that at most one arrow in $\mathscr{B}$ ends at $v$, too. Otherwise, if we assume that two different arrows $a$ and $a^{\prime}$ in $\mathscr{B}$ end at $v$, let $s(a)=u$ and $s\left(a^{\prime}\right)=u^{\prime}$ (clearly $u \neq u^{\prime}$, since at most one arrow starts at $u$ ), and let $q$ and $q^{\prime}$ be the maximal paths in $\mathscr{B}$ starting at $u$ and $u^{\prime}$, respectively. Then $q=a z$ and $q^{\prime}=a^{\prime} z^{\prime}$ for some paths $z$ and $z^{\prime}$ starting at $v$. But then $z$ and $z^{\prime}$ are subpaths of $p$, so one of them, say $z$, is a subpath of the other one. If $w=t(z)$, then $w=r(u)$, so $w \in L(C)$ and any path in $\mathscr{B}$ ending at $w$ is a subpath of $q=a z$. This provides a contradiction, since $a^{\prime} z$ is in $\mathscr{B}$ (as a subpath of $q^{\prime}$ ) and ends at $w$, but it is not a subpath of $q$.

We also have that if there is no arrow in $\mathscr{B}$ starting at a vertex $v$, then there is no arrow in $\mathscr{B}$ ending at $v$ either. Indeed, the maximal path in $\mathscr{B}$ starting at $v$ has length zero, so $r(v)=v$, and then $v \in L(C)$ and $l(v)=v$, which shows that no arrow in $\mathscr{B}$ ends at $v$.

Now taking the connected components of $C \cap \Gamma$ (regarded just as an undirected graph), and then considering the (directed) arrows, we find that $C \cap \Gamma=\bigsqcup_{i} \Gamma_{i}$ for some subquivers $\Gamma_{i}$ which can be of the types $\mathbb{A}_{\infty}, \mathbb{A}_{0, \infty}$ or $\mathbb{C}_{n}$, and this ends the proof.

Lemma 4.5. Let $C \subseteq K \Gamma$ be a left co-Frobenius path subcoalgebra. Let $u, v \in C \cap \Gamma$ be different vertices, and denote by $p_{u}$ and $p_{v}$ the maximal paths starting at $u$ and $v$, respectively. Then $p_{u}$ is not a subpath of $p_{v}$.

Proof. Assume the contrary. Then $p_{u}$ is a subpath of $p_{v}$. We know that $p_{u}$ and $p_{v}$ end at $r(u)$ and $r(v)$, respectively. Let $q$ be the subpath of $p_{v}$ which starts at $v$ and ends at $r(u)$. Since $p_{u}$ is a subpath of $p_{v}$, then $q$ contains $p_{u}$, too. Then both $q$ and $p_{u}$ end at $r(u)$, and since by Proposition $3.3 p_{u}$ is maximal with this property, we get $q=p_{u}$. This means that $u=v$ (as starting points of $p_{a}$ and $q$ ), a contradiction.

Now we are in the position to give the classification result for left co-Frobenius path subcoalgebras.

Theorem 4.6. Let $C$ be a path subcoalgebra of the path coalgebra $K \Gamma$, and let $B$ be a basis of paths of $C$. Then $C$ is left co-Frobenius if and only if $C \cap \Gamma=\bigsqcup_{i} \Gamma_{i}$, a disjoint union of subquivers of $\Gamma$ of one of types $\mathbb{A}_{\infty}, \mathbb{A}_{0, \infty}$ or $\mathbb{C}_{n}, n \geq 0$, and the path subcoalgebra $C_{i}$ of $K \Gamma_{i}$ spanned by the paths of $\mathcal{B}$ contained in $\Gamma_{i}$ is of type $K\left[\mathbb{A}_{\infty}, r\right]$ if $\Gamma_{i}=\mathbb{A}_{\infty}$, of type $K\left[\mathbb{A}_{0, \infty}, r\right]$ if $\Gamma_{i}=\mathbb{A}_{0, \infty}$, of type $K\left[\mathbb{C}_{n}, s\right]$ with $s \geq 1$ if $\Gamma_{i}=\mathbb{C}_{n}, n \geq 1$, and of type $K$ if $\Gamma_{i}=\mathbb{C}_{0}$. In this case $C=\bigoplus_{i} C_{i}$, in particular left co-Frobenius path subcoalgebras are direct sums of coalgebras of types $K\left[\mathbb{A}_{\infty}, r\right], K\left[\mathbb{A}_{0, \infty}, r\right], K\left[\mathbb{C}_{n}, s\right]$ or $K$.

Proof. By Lemma 4.4, $C \cap \Gamma=\bigsqcup_{i} \Gamma_{i}$, and any $\Gamma_{i}$ is of one of the types $\mathbb{A}_{\infty}, \mathbb{A}_{0, \infty}$ or $\mathbb{C}_{n}, n \geq 0$. Moreover, $C=\bigoplus_{i} C_{i}$, so $C$ is left co-Frobenius if and only if all $C_{i}$ 's are left co-Frobenius (see for example [15], Chapter 3). If all $C_{i}$ 's are of the indicated 
form, then they are left co-Frobenius by Examples 4.1, 4.2 and 4.3, and then so is $C$. Assume now that $C$ is left co-Frobenius. Then each $C_{i}$ is left co-Frobenius, so we can reduce to the case where $\Gamma$ is one of $\mathbb{A}_{\infty}, \mathbb{A}_{0, \infty}$ or $\mathbb{C}_{n}$, and $C \cap \Gamma=\Gamma$. As before, for each vertex $v$ we denote by $r(v)$ the end-point of the unique maximal path in $C$ starting at $v$, and by $p_{v}$ this maximal path. Also denote by $m(v)$ the length of $p_{v}$.

Case I. Let $\Gamma=\mathbb{C}_{n}$. If $n=0$, then $C \cong K$. If $n=1$, then $C \cong K\left[\mathbb{C}_{1}, s\right]$, since $m(\overline{0})=s>0$ because $\Gamma_{1} \subset C$, so there must be at least some nontrivial path in $C$. If $n \geq 2$, then $m(\bar{k}) \leq m(\overline{k+1})$ for any $\bar{k} \in \mathbb{Z}_{n}$, since otherwise $p_{\overline{k+1}}$ would be a subpath of $p_{\bar{k}}$, a contradiction by Lemma 4.5. Thus $m(\overline{0}) \leq m(\overline{1}) \leq$ $\cdots \leq m(\overline{n-1}) \leq m(\overline{0})$, so $m(\overline{0})=m(\overline{1})=\cdots=m(\overline{n-1})=m(\overline{0})=s$ for some $s \geq 0$. Since $C \cap \Gamma=\Gamma$, there are non-trivial paths in $C$, so $s>0$, and then clearly $C \cong K\left[\mathbb{C}_{n}, s\right]$.

Case II. If $\Gamma=\mathbb{A}_{\infty}$ or $\Gamma=\mathbb{A}_{0, \infty}$, then for any $n$ (in $\mathbb{Z}$ if $\Gamma=\mathbb{A}_{\infty}$, or in $\mathbb{N}$ if $\left.\Gamma=\mathbb{A}_{0, \infty}\right) m(n) \leq m(n+1)$ holds, otherwise $p_{n+1}$ would be a subpath of $p_{n}$, again a contradiction. Now if we take $r(n)=n+m(n)$ for any $n$, then $r$ is a strictly increasing function. Clearly $r(n)>n$, since $m(n)=0$ would contradict $C \cap \Gamma=\Gamma$. Now it is obvious that $C \cong K[\Gamma, r]$.

Corollary 4.7. Let $C \subseteq K \Gamma$ be a left and right co-Frobenius path subcoalgebra. Then $C$ is a direct sum of coalgebras of the type $K\left[\mathbb{A}_{\infty} \mid s\right], K\left[\mathbb{C}_{n}, s\right]$ or $K$.

Proof. It follows directly from Theorem 4.6 and the discussion at the end of each of Examples 4.1, 4.2 and 4.3, concerned to the property of being left and right coFrobenius.

Remark 4.8. (1) We have a uniqueness result for the representation of a left coFrobenius path subcoalgebras as a direct sum of coalgebras of the form $K\left[\mathbb{A}_{\infty}, r\right]$, $K\left[\mathbb{A}_{0, \infty}, r\right], K\left[\mathbb{C}_{n}, s\right]$ or $K$. To see this, an easy computation shows that the dual algebra of a coalgebra of any of these four types does not have non-trivial central idempotents, so it is indecomposable as an algebra. Now if $\left(C_{i}\right)_{i \in I}$ and $\left(D_{j}\right)_{j \in J}$ are two families of coalgebras with indecomposable dual algebras such that $\bigoplus_{i \in I} C_{i} \simeq$ $\bigoplus_{j \in J} D_{j}$ as coalgebras, then there is a bijection $\phi: J \rightarrow I$ such that $D_{j} \simeq C_{\phi(j)}$ for any $j \in J$. Indeed, if $f: \bigoplus_{i \in I} C_{i} \rightarrow \bigoplus_{j \in J} D_{j}$ is a coalgebra isomorphism, then the dual map $f^{*}: \prod_{j \in J} D_{j}^{*} \rightarrow \prod_{i \in I} C_{i}^{*}$ is an algebra isomorphism. Since all $C_{i}^{*}$ 's and $D_{j}^{*}$ 's are indecomposable, there is a bijection $\phi: J \rightarrow I$ and some algebra isomorphisms $\gamma_{j}: D_{j}^{*} \rightarrow C_{\phi(j)}^{*}$ for any $j \in J$, such that for any $\left(d_{j}^{*}\right)_{j \in J} \in$ $\prod_{j \in J} D_{j}^{*}$ the map $f^{*}$ takes $\left(d_{j}^{*}\right)_{j \in J}$ to the element of $\prod_{i \in I} C_{i}^{*}$ having $\gamma_{j}\left(d_{j}^{*}\right)$ on the $\phi(j)$-th slot. Regarding $C=\left(C_{i}\right)_{i \in I}$ as a left $C^{*}$-module and $D=\bigoplus_{j \in J} D_{j}$ as a left $D^{*}$-module in the usual way, with actions denoted by $\cdot$, the relation $f\left(f^{*}\left(d^{*}\right)\right.$. $c)=d^{*} \cdot f(c)$ holds for any $c \in C$ and $d^{*} \in D^{*}$. This shows that $f$ induces coalgebra isomorphisms $C_{\phi(j)} \simeq D_{j}$ for any $j \in J$. 
(2) The coalgebras of types $K\left[\mathbb{A}_{\infty}, r\right], K\left[\mathbb{A}_{0, \infty}, r\right], K\left[\mathbb{C}_{n}, s\right]$ or $K$ can be easily classified if we take into account that the sets of group-like elements are just the vertices and the non-trivial skew-primitives are scalar multiples of the arrows. There are no isomorphic coalgebras of two different types among these four types. Moreover:

(i) $K\left[\mathbb{A}_{\infty}, r\right] \simeq K\left[\mathbb{A}_{\infty}, r^{\prime}\right]$ if and only if there is an integer $h$ such that $r^{\prime}(n)=$ $r(n+h)$ for any integer $n$;

(ii) $K\left[\mathbb{A}_{0, \infty}, r\right] \simeq K\left[\mathbb{A}_{0, \infty}, r^{\prime}\right]$ if and only if $r=r^{\prime}$;

(iii) $K\left[\mathbb{C}_{n}, s\right] \simeq K\left[\mathbb{C}_{m}, s^{\prime}\right]$ if and only $n=m$ and $s=s^{\prime}$.

\section{Examples}

It is known (see [24], [12]) that any pointed coalgebra can be embedded in a path coalgebra. Thus it is expected that there is a large variety of co-Frobenius subcoalgebras of path coalgebras if we do not restrict only to the class of path subcoalgebras. The aim of this section is to provide several such examples. We first explain a simple construction connecting incidence coalgebras and path coalgebras, and producing examples as we wish.

As a pointed coalgebra, any incidence coalgebra can be embedded in a path coalgebra. However, there is a more simple way to define such an embedding for incidence coalgebras than for arbitrary pointed coalgebras. Indeed, let $X$ be a locally finite partially ordered set. Consider the quiver $\Gamma$ with vertices the elements of $X$, and such that there is an arrow from $x$ to $y$ if and only if $x<y$ and there is no element $z$ with $x<z<y$. With this notation, it is an easy computation to check the following.

Proposition 5.1. The linear map $\phi: K X \rightarrow K \Gamma$, defined by

$$
\phi\left(e_{x, y}\right)=\sum_{\substack{p \text { path } \\ s(p)=x, t(p)=y}} p
$$

for any $x, y \in X, x \leq y$, is an injective coalgebra morphism.

Note that in the previous proposition $\phi(K X)$ is in general a subcoalgebra of $K \Gamma$ which is not a path subcoalgebra. This suggests that when we deal with left coFrobenius subcoalgebras of incidence coalgebras, which of course embed themselves in $K \Gamma$ (usually not as path subcoalgebras), structures that are more complicated than those of left co-Frobenius path subcoalgebras can appear. Thus the classification of left co-Frobenius subcoalgebras of incidence coalgebras is probably more difficult. The next example is evidence in this direction.

Example 5.2. Let $s \geq 2$ and $X=\left\{a_{n} \mid n \in \mathbb{Z}\right\} \cup\left(\bigcup_{n \in \mathbb{Z}}\left\{b_{n, i} \mid 1 \leq i \leq s\right\}\right)$ with the ordering $\leq$ such that $a_{n}<b_{n, i}<a_{n+1}$ for any integer $n$ and any $1 \leq i \leq s$, and $b_{n, i}$ and $b_{n, j}$ are not comparable for any $n$ and $i \neq j$.

Let $C$ be the subcoalgebra of $K X$ spanned by the following elements: 
- the elements $e_{x, x}, x \in X$,

- all segments $e_{x, y}$ of length 1 ,

- the segments $e_{a_{n}, a_{n+1}}, n \in \mathbb{Z}$,

- the segments $e_{b_{n, i}, b_{n+1, i}}$, with $n \in \mathbb{Z}$ and $1 \leq i \leq s$.

Then by applying Proposition 3.3, we see that $C$ is co-Frobenius.

If we take the subcoalgebra $D$ of $C$ obtained by restricting to the non-negative part of $X$, i.e., $D$ is spanned by the elements $e_{x, y}$ in the indicated basis of $C$ with both $x$ and $y$ among $\left\{a_{n} \mid n \geq 0\right\} \cup\left(\bigcup_{n \geq 0}\left\{b_{n, i} \mid 1 \leq i \leq s\right\}\right)$, we see that $D$ is left co-Frobenius, but not right co-Frobenius.

Now let $\Gamma$ be the quiver associated to the ordered set $X$ as in the discussion above.
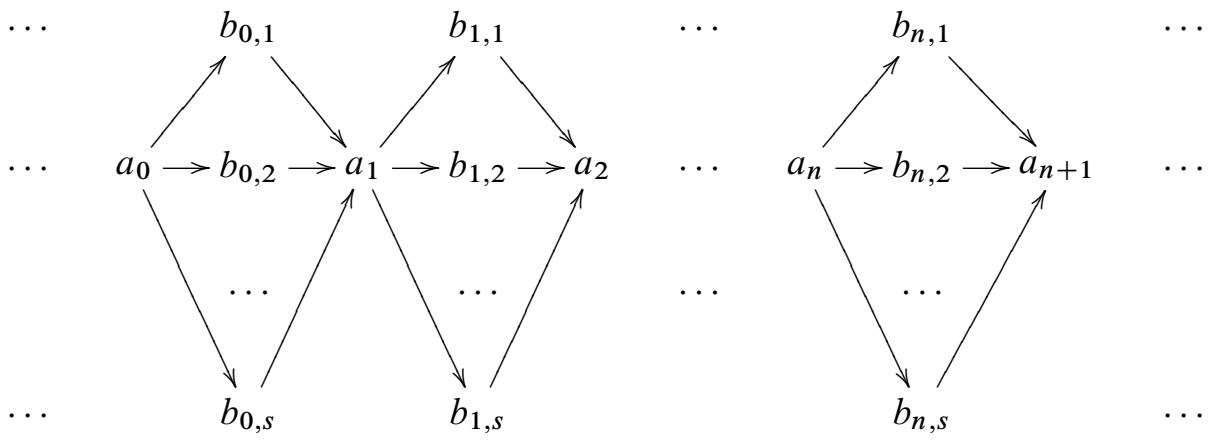

If $\phi: K X \rightarrow K \Gamma$ is the embedding described in Proposition 5.1, then $\phi(C)$ is a co-Frobenius subcoalgebra of $K \Gamma$. We see that $\phi(C)$ is the subspace of $K \Gamma$ spanned by the vertices of $\Gamma$, the paths of length 1 , the paths $\left[b_{n, i} a_{n+1} b_{n+1, i}\right]$ with $n \in \mathbb{Z}$ and $1 \leq i \leq s$, and the elements $\sum_{1 \leq i \leq s}\left[a_{n} b_{n, i} a_{n+1}\right]$ with $n \in \mathbb{Z}$, thus $\phi(C)$ is not a path subcoalgebra. Here we denote by $\left[b_{n, i} a_{n+1} b_{n+1, i}\right]$ and $\left[a_{n} b_{n, i} a_{n+1}\right]$ the paths following the indicated vertices and the arrows between them. By restricting to the non-negative part of $X$, a similar description can be given for $\phi(D)$, a subcoalgebra of $K \Gamma$ which is left co-Frobenius but not right co-Frobenius.

It is possible to embed some of the co-Frobenius path subcoalgebras in other path coalgebras as subcoalgebras which are not path subcoalgebras.

Example 5.3. Consider the quiver $\mathbb{A}_{\infty}$ with vertices indexed by the integers, with the path from $i$ to $j$ denoted by $p_{i, j}$. Consider the path subcoalgebra $D=K\left[\mathbb{A}_{\infty} \mid 2\right]$, with basis $\left\{p_{i, i}, p_{i, i+1}, p_{i, i+2} \mid i \in \mathbb{Z}\right\}$. We also consider the quiver $\Gamma$
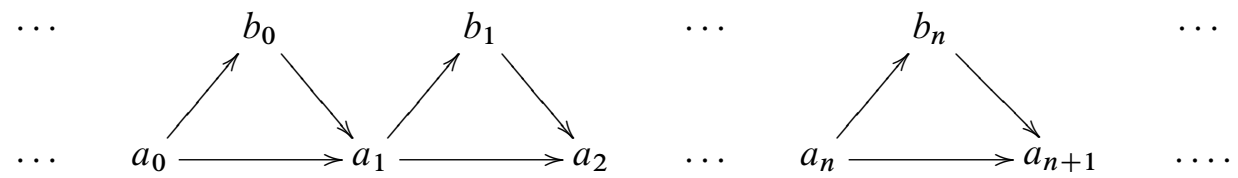
Then $\mathbb{A}_{\infty}$ is a subquiver of $\Gamma$ if we identify $a_{i}$ with $2 i$ and $b_{i}$ with $2 i+1$ for any integer $i$. Thus $K \mathbb{A}_{\infty}$ is a subcoalgebra of $K \Gamma$ in the obvious way, and then so is $D$. However, there is another way to embed $D$ in $K \Gamma$. Indeed, the linear map $\phi: D \rightarrow K \Gamma$ defined by

$$
\begin{aligned}
\phi\left(p_{2 i, 2 i}\right) & =a_{i}, \\
\phi\left(p_{2 i+1,2 i+1}\right) & =b_{i}, \\
\phi\left(p_{2 i, 2 i+1}\right) & =\left[a_{i} b_{i}\right], \\
\phi\left(p_{2 i+1,2 i+2}\right) & =\left[b_{i} a_{i+1}\right], \\
\phi\left(p_{2 i, 2 i+2}\right) & =\left[a_{i} a_{i+1}\right]+\left[a_{i} b_{i} a_{i+1}\right], \\
\phi\left(p_{2 i+1,2 i+3}\right) & =\left[b_{i} a_{i+1} b_{i+1}\right]
\end{aligned}
$$

for any $i \in \mathbb{Z}$ is an injective morphism of coalgebras. Here we denote by $\left[a_{i} b_{i}\right]$, $\left[a_{i} b_{i} a_{i+1}\right]$, etc., the paths following the respective vertices and arrows. We conclude that the subcoalgebra $C=\phi(D)$ of $K \Gamma$, spanned by all vertices $a_{n}, b_{n}$, all arrows $\left[a_{n} a_{n+1}\right],\left[a_{n} b_{n}\right],\left[b_{n} a_{n+1}\right]$ and the elements $\left[a_{n} b_{n} a_{n+1}\right]+\left[a_{n} a_{n+1}\right]$ and $\left[b_{n} a_{n+1} b_{n+1}\right]$, is co-Frobenius. Note that $D$ is not a path subcoalgebra of $K \Gamma$. This can be also seen as the subcoalgebra of the incidence coalgebra of $\mathbb{Z}$ with basis consisting of segments of length at most 2 .

Note that in the above example, we can also consider a similar situation but with all segments $e_{n, n+i}$ of the incidence coalgebra of $\mathbb{Z}$ which have length less or equal to a certain positive integer $s(i \leq s)$; the same properties as above would then hold for this situation.

Example 5.4. We consider the same situation as above, but we restrict the quiver $\Gamma$ to the non-negative part:
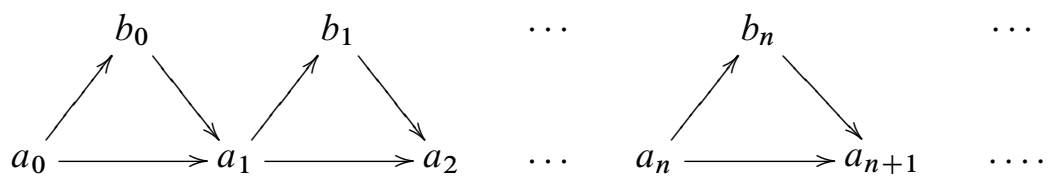

Equivalently, we consider the subcoalgebra of the incidence coalgebra of $\mathbb{N}$ with a basis of all segments of length less or equal to 2 (or $\leq s$ for more generality). This coalgebra is left co-Frobenius but not right co-Frobenius, it is a subcoalgebra of an incidence coalgebra, and it can also be regarded as a subcoalgebra of a path coalgebra, but without a basis of paths.

Now we prove a simple, but useful result, which shows that the category of incidence coalgebras is closed under tensor product of coalgebras.

Proposition 5.5. Let $X, Y$ be locally finite partially ordered sets. Consider on $X \times Y$ the order $(x, y) \leq\left(x^{\prime}, y^{\prime}\right)$ if and only if $x \leq y$ and $x^{\prime} \leq y^{\prime}$. Then there is an isomorphism of coalgebras $K(X \times Y) \cong K X \otimes K Y$. 
Proof. It is clear that $X \times Y$ is locally finite. We show that the natural isomorphism of vector spaces $\varphi: K(X \times Y) \rightarrow K X \otimes K Y, \varphi\left(e_{(x, y),\left(x^{\prime}, y^{\prime}\right)}\right)=e_{x, x^{\prime}} \otimes e_{y, y^{\prime}}$, is a morphism of coalgebras. This is well defined by the definition of the order relation on $X \times Y$. For comultiplication we have

$$
\begin{aligned}
& \sum \varphi\left(e_{(x, y),\left(x^{\prime}, y^{\prime}\right)}\right)_{1} \otimes\left(e_{\left.(x, y),\left(x^{\prime}, y^{\prime}\right)\right)_{2}=} \sum_{x \leq a \leq x^{\prime}} \sum_{y \leq b \leq y^{\prime}} e_{x, a} \otimes e_{y, b} \otimes e_{a, x^{\prime}} \otimes e_{x^{\prime}, b}\right. \\
& =\sum_{\substack{(x, y) \leq(a, b) \\
\leq\left(x^{\prime}, y^{\prime}\right)}} \varphi\left(e_{(x, y),(a, b)}\right) \otimes \varphi\left(e_{(a, b),\left(x^{\prime}, y^{\prime}\right)}\right)
\end{aligned}
$$

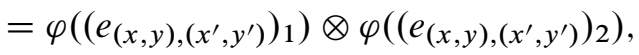

and it is also easy to see that $\varepsilon_{K X \otimes K Y} \circ \varphi=\varepsilon_{K(X \times Y)}$.

Example 5.6. Consider the ordered set $(\mathbb{Z} \times \mathbb{Z}, \leq)$, with order given by the direct product of the orders of $(\mathbb{Z}, \leq)$ and $(\mathbb{Z}, \leq)$. Thus $(i, j) \leq(p, q)$ if and only if $i \leq p$ and $j \leq q$. We know from Proposition 5.5 that $\psi: K \mathbb{Z} \otimes K \mathbb{Z} \rightarrow K(\mathbb{Z} \times \mathbb{Z})$, $\psi\left(e_{i, p} \otimes e_{j, q}\right)=e_{(i, j),(p, q)}$, is an isomorphism of coalgebras.

With the notation preceding Proposition 5.1, the quiver $\Gamma$ associated to the locally finite ordered set $(\mathbb{Z} \times \mathbb{Z}, \leq)$ is

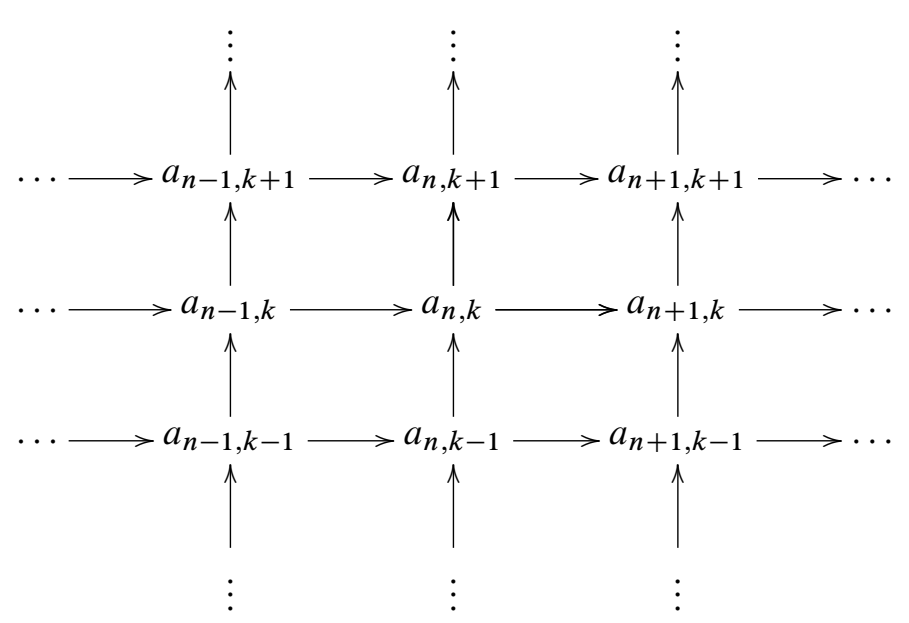

where we just denoted the vertices by $a_{n, k}$ instead of just $(n, k)$. Let $\phi: K(\mathbb{Z} \times$ $\mathbb{Z}) \rightarrow K \Gamma$ be the embedding from Proposition 5.1. If we consider the subcoalgebra $K\left[\mathbb{A}_{\infty} \mid 1\right]$ of $K \mathbb{Z}$, then $K\left[\mathbb{A}_{\infty} \mid 1\right] \otimes K\left[\mathbb{A}_{\infty} \mid 1\right]$ is a subcoalgebra of $K \mathbb{Z} \otimes K \mathbb{Z}$, so then $C=\phi \psi\left(K\left[\mathbb{A}_{\infty} \mid 1\right] \otimes K\left[\mathbb{A}_{\infty} \mid 1\right]\right)$, which is the subspace spanned by the vertices of $\Gamma$, the arrows of $\Gamma$, and the elements $\left[a_{n, k} a_{n+1, k} a_{n+1, k+1}\right]+\left[a_{n, k} a_{n, k+1}, a_{n+1, k+1}\right]$, is a subcoalgebra of $K \Gamma$. Since $K\left[\mathbb{A}_{\infty} \mid 1\right]$ is co-Frobenius, and the tensor product of co-Frobenius coalgebras is co-Frobenius (see [21], Proposition 4.15), we obtain that $C$ is a co-Frobenius coalgebra. Alternatively, it can be seen that $\psi\left(K\left[\mathbb{A}_{\infty} \mid 1\right] \otimes\right.$ $\left.K\left[\mathbb{A}_{\infty} \mid 1\right]\right)$, which is the subspace spanned by the elements $e_{(n, k),(n, k)}, e_{(n, k),(n+1, k)}$, 
$e_{(n, k),(n, k+1)}, e_{(n, k),(n+1, k+1)}$ with arbitrary $n, k \in \mathbb{Z}$, is co-Frobenius by applying Proposition 3.3. $C$ can be seen as both a subcoalgebra of an incidence coalgebra and of a path coalgebra, but not with a basis of paths. We note that $C$ is not even isomorphic to a path subcoalgebra. Indeed, if it were so, it should be isomorphic to some $K\left[\mathbb{A}_{\infty} \mid s\right]$, since it is infinite dimensional and indecomposable. But in $C$, for any group-like element $g$ there are precisely two other group-like elements $h$ with the property that the set of non-trivial $(h, g)$-skew-primitive elements is nonempty, while for any group-like element $\mathrm{g}$ of $K\left[\mathbb{A}_{\infty} \mid s\right]$ there is only one such $h$.

With similar arguments, we can give a more general version of the previous example, by considering finite tensor products of coalgebras of type $K\left[\mathbb{A}_{\infty} \mid s\right]$, as follows.

Example 5.7. Let $D=K\left[\mathbb{A}_{\infty} \mid s_{1}\right] \otimes K\left[\mathbb{A}_{\infty} \mid s_{2}\right] \otimes \cdots \otimes K\left[\mathbb{A}_{\infty} \mid s_{m}\right]$, where $m \geq 2$ and $s_{1}, \ldots, s_{m}$ are positive integers. Then $D$ is co-Frobenius as a tensor product of co-Frobenius coalgebras, and $D$ embeds in the $m$-fold tensor product $K \mathbb{Z} \otimes K \mathbb{Z} \otimes$ $\cdots \otimes K \mathbb{Z}$. But this last tensor product is isomorphic to the incidence coalgebra of the ordered set $\mathbb{Z}^{m}=\mathbb{Z} \times \mathbb{Z} \times \cdots \times \mathbb{Z}$, with the direct product order. The image of $D$ via this embedding is the subcoalgebra $E$ of $K(\mathbb{Z} \times \mathbb{Z} \times \cdots \times \mathbb{Z})$ spanned by all the segments $e_{\left(n_{1}, \ldots, n_{m}\right),\left(k_{1}, \ldots, k_{m}\right)}$ with $n_{1} \leq k_{1} \leq n_{1}+s_{1}, \ldots, n_{m} \leq k_{m} \leq n_{m}+s_{m}$.

Now if we consider the quiver $\Gamma$ associated to the ordered set $\mathbb{Z} \times \mathbb{Z} \times \cdots \times \mathbb{Z}$ as in the beginning of this section, we have an embedding of $K(\mathbb{Z} \times \mathbb{Z} \times \cdots \times \mathbb{Z})$ in $K \Gamma$. Denote the vertices of $\Gamma$ by $a_{n_{1}, \ldots, n_{m}}$. The image of $E$ through this embedding is the subcoalgebra $C$ of $K \Gamma$ spanned by all the elements of the form $S\left(\Gamma,\left(n_{1}, \ldots, n_{m}\right),\left(k_{1}, \ldots, k_{m}\right)\right)$, with $n_{1}, \ldots, n_{m}, k_{1}, \ldots, k_{m}$ integers such that $n_{1} \leq k_{1} \leq n_{1}+s_{1}, \ldots, n_{m} \leq k_{m} \leq n_{m}+s_{m}$, where we denote the sum of all paths in $\Gamma$ starting at $a_{n_{1}, \ldots, n_{m}}$ and ending at $a_{k_{1}, \ldots, k_{m}}$ by $S\left(\Gamma,\left(n_{1}, \ldots, n_{m}\right),\left(k_{1}, \ldots, k_{m}\right)\right)$. Thus $C$ is a co-Frobenius subcoalgebra of $K \Gamma$, which is also isomorphic to a subcoalgebra of an incidence coalgebra. However, $C$ is not a path subcoalgebra, and not even isomorphic to a path subcoalgebra. Indeed, for any group-like element $g$ of $E$ there are precisely $m$ group-like elements $h$ for which there are non-trivial $(h, g)$-skew-primitive elements, while in a co-Frobenius path subcoalgebra for any group-like element $g$ there is at most one such $h$.

Remark 5.8. We note that the co-Frobenius coalgebra $C$ constructed in Example 5.2 is not isomorphic to a coalgebra of the form $K\left[\mathbb{A}_{\infty} \mid s_{1}\right] \otimes K\left[\mathbb{A}_{\infty} \mid s_{2}\right] \otimes \cdots \otimes K\left[\mathbb{A}_{\infty} \mid s_{m}\right]$. Indeed, if $g=b_{n, i}$ there exists exactly one group-like element $h$ of $C$ such that there are non-trivial $(h, g)$-skew-primitive elements (this is $h=a_{n+1}$ ), and if $g=a_{n}$ there exist $s$ such group-like elements $h$ (these are $b_{n, 1}, \ldots, b_{n, s}$ ). On the other hand, in $K\left[\mathbb{A}_{\infty} \mid s_{1}\right] \otimes K\left[\mathbb{A}_{\infty} \mid s_{2}\right] \otimes \cdots \otimes K\left[\mathbb{A}_{\infty} \mid s_{m}\right]$ for any group-like element $g$ there exist precisely $m$ such elements $h$.

We end with another explicit example, which shows that there are co-Frobenius 
subcoalgebras of path coalgebras that are isomorphic neither to a path subcoalgebra nor to a subcoalgebra of an incidence coalgebra.

Example 5.9. Let $\Gamma$ be the graph

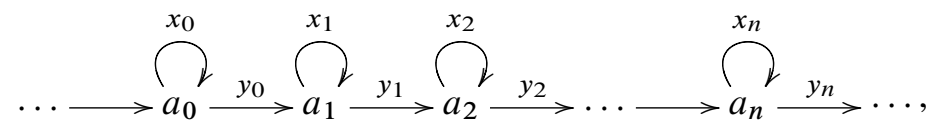

and let $C$ be the subcoalgebra of the path coalgebra of $\Gamma$ having a basis the elements $a_{n}, x_{n}, y_{n}$ and $y_{n}+x_{n} y_{n}$. This is, in fact, isomorphic to $K\left[\mathbb{C}_{1} \mid 1\right] \otimes K\left[\mathbb{A}_{\infty} \mid 1\right]$, so it is co-Frobenius. By the classification theorem for co-Frobenius path subcoalgebras and the structure of the skew-primitive elements of $C$, we see that $C$ is not isomorphic to a path subcoalgebra. We note that it is not isomorphic either to a subcoalgebra of an incidence coalgebra because in an incidence coalgebra if $g$ is any group-like element, there is no $(g, g)$ - skew-primitive element, while in $C$ for each group-like $g=a_{n}$, $x_{n}$ is a $(g, g)$-skew-primitive.

\section{Hopf algebra structures on path subcoalgebras}

In this section we discuss the possibility of extending the coalgebra structure of a path subcoalgebra to a Hopf algebra structure. First of all, it is a simple application of Proposition 3.4 to see when a finite dimensional path coalgebra has a Hopf algebra structure.

Proposition 6.1. If the path coalgebra $K \Gamma$ is finite dimensional, then it has a Hopf algebra structure if and only if it is cosemisimple, i.e., $\Gamma$ has no arrows.

Proof. If the finite dimensional $K \Gamma$ has a Hopf algebra structure, then it has nonzero integrals, so it is left (and right) co-Frobenius, and $K \Gamma$ is cosemisimple by Proposition 3.4. Conversely, if there are no arrows, then $K \Gamma$ can be endowed with the group Hopf algebra structure obtained if we consider a group structure on the set of vertices.

Next, we are interested in finding examples of Hopf algebra structures that can be defined on some path subcoalgebras. At this point we discuss only cases where the resulting Hopf algebra has non-zero integrals, i.e., it is left (or right) co-Frobenius. Thus the path subcoalgebras that we consider are among the ones in Corollary 4.7. We ask the following general question.

Problem. Which of the left and right co-Frobenius path subcoalgebras (classified in Corollary 4.7) can be endowed with a Hopf algebra structure? 
In the rest of this section we solve the problem in the case where $K$ is a field containing primitive roots of unity of any positive order, in particular $K$ has characteristic zero. We will make this assumption on $K$ from this point on. We just note that some of the constructions can be also done in positive characteristic, if we just require that $K$ contains certain primitive roots of unity and the characteristic of $K$ is large enough.

Proposition 6.2. (I) Let $s>0$ be an integer. Let $q$ be a primitive $(s+1)$-th root of unity in $K$. Let $G$ be a group such that there exist an element $g \in Z(G)$ of infinite order and a character $\chi \in G^{*}$ such that $\chi(g)=q$. Also let $\alpha \in K$ which may be non-zero only if $\chi^{s+1}=1$. Consider the algebra generated by the elements of $G$ (and preserving the group multiplication on these elements) and $x$ subject to relations

$$
x h=\chi(h) h x \text { for any } h \in G, \quad x^{s+1}=\alpha\left(g^{s+1}-1\right)
$$

(that is, the free or amalgamated product $K[x] * K[G]$, factored out by the above relations). Then this algebra has a unique Hopf algebra structure such that the elements of $G$ are group-like elements, $\Delta(x)=1 \otimes x+x \otimes g$, and $\varepsilon(x)=0$. We denote this Hopf algebra structure by $H_{\infty}(s, q, G, g, \chi, \alpha)$.

(II) Let $n \geq 2$ and $s>0$ be integers such that $s+1$ divides $n$. Let $q$ be a primitive $(s+1)$-th root of unity in $K$. Consider a group $G$ such that there exist an element $g \in Z(G)$ of order $n$ and a character $\chi \in G^{*}$ such that $\chi(g)=q$. Also let $\alpha \in K$ which may be non-zero only if $\chi^{s+1}=1$. Consider the algebra generated by the elements of $G$ (and preserving the group multiplication on these elements) and $x$ subject to relations

$$
x h=\chi(h) h x \text { for any } h \in G, \quad x^{s+1}=\alpha\left(g^{s+1}-1\right) .
$$

Then this algebra has a unique Hopf algebra structure such that the elements of $G$ are group-like elements, $\Delta(x)=1 \otimes x+x \otimes g$, and $\varepsilon(x)=0$. We denote this Hopf algebra structure by $H_{n}(s, q, G, g, \chi, \alpha)$.

Proof. We consider an approach similar to the one in [4]. For both (I) and (II) we consider the Hopf group algebra $K G$, and its Ore extension $K G[X, \bar{\chi}]$, where $\bar{\chi}$ is the algebra automorphism of $K G$ such that $\bar{\chi}(h)=\chi(h) h$ for any $h \in G$. Since $g \in Z(G)$, this Ore extension has a unique Hopf algebra structure such that $\Delta(X)=1 \otimes X+X \otimes g$ and $\varepsilon(X)=0$, by the universal property for Ore extensions (see for example [4], Lemma 1.1). Since $(1 \otimes X)(X \otimes g)=q(X \otimes g)(1 \otimes X)$, the quantum binomial formula shows that $\Delta\left(X^{s+1}\right)=1 \otimes X^{s+1}+X^{s+1} \otimes g^{s+1}$, so the ideal $I=\left(X^{s+1}-\alpha\left(g^{s+1}-1\right)\right)$ is in fact a Hopf ideal of $K G[X, \bar{\chi}]$. Then we can consider the factor Hopf algebra $K G[X, \bar{\chi}] / I$, and this is just the desired Hopf algebra $H_{\infty}(s, q, G, g, \chi, \alpha)$ in case (I) and $H_{n}(s, q, G, g, \chi, \alpha)$ in case (II). The condition that $\alpha=0$ whenever $\chi^{s+1} \neq 1$ guarantees that the map $G \rightarrow K G[X, \bar{\chi}] / I$ taking an element $h \in G$ to its class modulo $I$ is injective, thus $G$ is the group of group-like elements of this factor Hopf algebra. 
In the following example we give examples of co-Frobenius path subcoalgebras that can be endowed with Hopf algebra structures. Moreover, we don't only introduce one such structure, but a family of Hopf algebra structures on each path subcoalgebra considered in the example.

Example 6.3. (i) $K\left[\mathbb{A}_{\infty} \mid s\right]$ can be endowed with a Hopf algebra structure for any $s \geq 1$. Indeed, let $q$ be a primitive $(s+1)$-th root of unity in $\mathrm{K}$, and let $\alpha \in K$. We define a multiplication (on basis elements, then extended linearly) on $K\left[\mathbb{A}_{\infty} \mid s\right]$ by

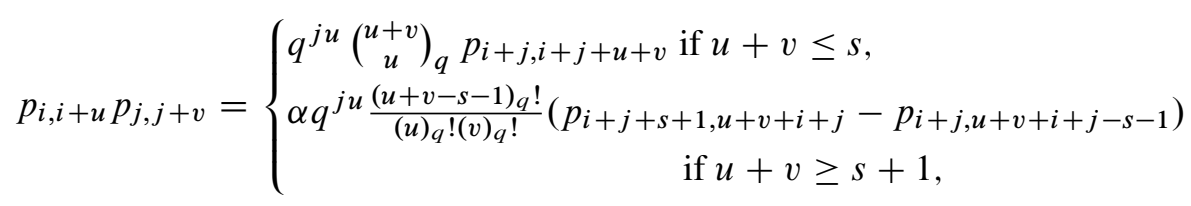

where $\left(\begin{array}{c}u+v \\ u\end{array}\right)_{q}$ denotes the $q$-binomial coefficient. Then this multiplication makes $K\left[\mathbb{A}_{\infty} \mid s\right]$ an algebra, which together the initial coalgebra structure define a Hopf algebra structure on $K\left[\mathbb{A}_{\infty} \mid s\right]$. Indeed, we can see this by considering the Hopf algebra $H_{\infty}\left(s, q, C_{\infty}, c, \chi, \alpha\right)$, where $C_{\infty}$ is the (multiplicative) infinite cyclic group generated by an element $c$, and the character $\chi$ is defined by $\chi(c)=q$. Thus $H_{\infty}\left(s, q, C_{\infty}, c, \chi, \alpha\right)$ is generated as an algebra by the elements $c$ and $x$, subject to relations $x c=q c x$ and $x^{s+1}=\alpha\left(c^{s+1}-1\right)$, and with coalgebra structure such that $\Delta(c)=c \otimes c, \varepsilon(c)=1$, and $\Delta(x)=1 \otimes x+x \otimes c$. Since $(1 \otimes x)(x \otimes c)=$ $q(x \otimes c)(1 \otimes x)$, we can apply the quantum binomial formula and get

$$
\Delta\left(x^{u}\right)=\sum_{0 \leq h \leq u}\left(\begin{array}{l}
u \\
h
\end{array}\right)_{q} x^{u-h} \otimes c^{u-h} x^{h}
$$

and then

$$
\Delta\left(\frac{1}{(u)_{q} !} c^{i} x^{u}\right)=\sum_{0 \leq h \leq u} \frac{1}{(u-h)_{q} !} c^{i} x^{u-h} \otimes \frac{1}{(h)_{q} !} c^{i+u-h} x^{h}
$$

for any $0 \leq u \leq s$ and any integer $i$. Therefore if we denote $\frac{1}{(u)_{q} !} c^{i} x^{u}$ by $P_{i, i+u}$, this means that $\Delta\left(P_{i, i+u}\right)=\sum_{0 \leq h \leq u} P_{i, i+h} \otimes P_{i+h, i+u}$, showing that the linear isomorphism $\phi: K\left[\mathbb{A}_{\infty} \mid s\right] \rightarrow H_{\infty}\left(s, q, C_{\infty}, c, \chi, \alpha\right)$ taking $p_{i, i+u}$ to $P_{i, i+u}$ for any $0 \leq u \leq s$ and $i \in \mathbb{Z}$ is an isomorphism of coalgebras. Now we just transfer the algebra structure of $H_{\infty}\left(s, q, C_{\infty}, c, \chi, \alpha\right)$ through $\phi^{-1}$ and get precisely the multiplication formula given above.

(ii) Let us consider now the coalgebra $C$ which is a direct sum of a family of copies of (the same) $K\left[\mathbb{A}_{\infty} \mid S\right]$, indexed by a non-empty set $P$. Then $C$ can be endowed with a Hopf algebra structure. To see this, we extend the example from (i) as follows. Let $G$ be a group such that there exist an element $g \in Z(G)$ of infinite order and a character $\chi \in G^{*}$ for which $q=\chi(g)$ is a primitive $(s+1)$-th root of unity, and moreover the factor group $G /\langle g\rangle$ is in bijection with the set $P$ (note that such a triple $(G, g, \chi)$ 
always exists; we can take for instance a group structure on the set $P, G=C_{\infty} \times P$, $g$ a generator of $C_{\infty}$, and $\chi$ defined such that $\chi(g)=q$ and $\chi(p)=1$ for any $\left.p \in P\right)$. For simplicity of the notation, we can assume that $P$ is a set of representatives for the $\langle g\rangle$-cosets of G. Consider the Hopf algebra $A=H_{\infty}(s, q, G, g, \chi, \alpha)$, where $\alpha$ is a scalar which may be non-zero only if $\chi^{s+1}=1$. Then the subalgebra $B$ of $A$ generated by $g$ and $x$ is a Hopf subalgebra isomorphic to $K\left[\mathbb{A}_{\infty} \mid s\right]$ as a coalgebra, and $A=\bigoplus_{p \in P} p B$ is a direct sum of subcoalgebras, all isomorphic to $K\left[\mathbb{A}_{\infty} \mid s\right]$. Thus $A$ is isomorphic as a coalgebra to $C$, and we can transfer the Hopf algebra structure of $A$ to $C$.

(iii) Assume that $n \geq 2$ and $s+1$ divides $n$. Then $K\left[\mathbb{C}_{n}, s\right]$ can be endowed with a Hopf algebra structure. Indeed, we proceed as for $K\left[\mathbb{A}_{\infty} \mid s\right]$, but replacing the Hopf algebra $H_{\infty}\left(s, q, C_{\infty}, c, \chi, \alpha\right)$ by $H_{n}\left(s, q, C_{n}, c, \chi, \alpha\right)$, where $C_{n}$ is a cyclic group of order $n$ with a generator $c$ (we have the same relations for $c$ and $x$ as in (i), to which we add $c^{n}=1$ ). Thus the multiplication of $K\left[\mathbb{A}_{\infty} \mid s\right]$ is given by

$$
q_{\bar{i} \mid u} q_{\bar{j} \mid v}=\left\{\begin{array}{c}
q^{j u}\left(\begin{array}{c}
u+v \\
u
\end{array}\right)_{q} q_{\overline{i+j}} \mid u+v \\
\left.\alpha q^{j u \frac{(u+v-s-1)_{q} !}{(u)_{q} !(v)_{q} !}\left(q_{\overline{i+j+s+1}} \mid u+v-s-1\right.}-q_{\overline{i+j} \mid u+v-s-1}\right) \\
\text { if } u+v \geq s+1 .
\end{array}\right.
$$

Also, as in (ii), a direct sum of copies of the same $K\left[\mathbb{C}_{n}, s\right]$, indexed by an arbitrary non-empty set $P$, can be endowed with a Hopf algebra structure isomorphic to some $H_{n}(s, q, G, g, \chi, \alpha)$ for some $q, G, g, \chi, \alpha$, where $q$ is a primitive $(s+1)$-th root of unity, $G$ is a group, $g \in Z(G)$ is an element of order $n, G /\langle g\rangle$ is in bijection with $P, \chi \in G^{*}$ is a character such that $\chi(g)=q$, and $\alpha \in K$ is a scalar which may be non-zero only if $\chi^{s+1}=1$.

The examples given in (iii) appear (for finite sets $P$ ) in [10].

Now we can prove the main result of this section.

Theorem 6.4. Assume that $K$ is a field containing primitive roots of unity of any positive order (in particular, $K$ has characteristic 0 ). Then a co-Frobenius path subcoalgebra $C \neq 0$ can be endowed with a Hopf algebra structure if and only if it is of one of the following three types:

(I) A direct sum of copies (indexed by a set $P$ ) of the same $K\left[\mathbb{A}_{\infty} \mid S\right]$ for some $s \geq 1$. In this case, any Hopf algebra structure on $C$ is isomorphic to a Hopf algebra of the form $H_{\infty}(s, q, G, g, \chi, \alpha)$ for some $q, G, g, \chi, \alpha$, where $q$ is a primitive $(s+1)$ th root of unity, $G$ is a group, $g \in Z(G)$ is an element of infinite order, $G /\langle g\rangle$ is in bijection with $P, \chi \in G^{*}$ is a character such that $\chi(g)=q$, and $\alpha \in K$ is a scalar which may be non-zero only if $\chi^{s+1}=1$.

(II) A direct sum of copies (indexed by a set $P$ ) of the same $K\left[\mathbb{C}_{n}, s\right]$ for some $n \geq 2$ and $s \geq 1$ such that $s+1$ divides $n$. In this case, any Hopf algebra structure on $C$ is isomorphic to a Hopf algebra of the form $H_{n}(s, q, G, g, \chi, \alpha)$ for some $q, G$, $g, \chi, \alpha$, where $q$ is a primitive $(s+1)$-th root of unity, $G$ is a group, $g \in Z(G)$ is 
an element of order $n, G /\langle g\rangle$ is in bijection with $P, \chi \in G^{*}$ is a character such that $\chi(g)=q$, and $\alpha \in K$ is a scalar which may be non-zero only if $\chi^{s+1}=1$.

(III) A direct sum of copies of $K$. In this case, any Hopf algebra structure on $C$ is isomorphic to a group Hopf algebra $K G$ for some group $G$.

Proof. By Example 6.3 we see that a coalgebra of type (I) or (II) has a Hopf algebra structure. Obviously, a coalgebra of type (III) is a group-like coalgebra $K X$ for some set $X$, so then it has a Hopf algebra structure, obtained if we endow $X$ with a group structure.

Conversely, let $C$ be a co-Frobenius path subcoalgebra which can be endowed with a Hopf algebra structure. By Corollary 4.7, $C$ is isomorphic to a direct sum of coalgebras of types $K\left[\mathbb{A}_{\infty} \mid s\right], K\left[\mathbb{C}_{n}, s\right]$ or $K$. We have that $G=G(C)$, the set of all vertices of $C$, is a group with the induced multiplication. We look at the identity element 1 of this group and distinguish three cases.

Case 1. If 1 is a vertex in a connected component of type $K\left[\mathbb{A}_{\infty} \mid s\right]$, denote the vertices of this connected component by $\left(v_{n}\right)_{n \in \mathbb{Z}}$ such that $v_{0}=1$. Also denote by $a_{n}$ the arrow from $v_{n}$ to $v_{n+1}$ for any $n \in \mathbb{Z}$. If $g=v_{1}$, then $\Delta\left(a_{1}\right)=1 \otimes a_{1}+a_{1} \otimes g$ and $a_{1} \notin C_{0}$. Then $\Delta\left(g a_{1}\right)=g \otimes g a_{1}+g a_{1} \otimes g^{2}$, and $g a_{1} \notin C_{0}$, so $P_{g^{2}, g}(C) \nsubseteq C_{0}$. Since the only $h \in G$ such that $P_{h, g}(C)$ is not trivial (i.e., $\neq K(h-g)$, or equivalently, not contained in $C_{0}$ ) is $h=v_{2}$, we obtain $v_{2}=g^{2}$. Recurrently we see that $v_{n}=g^{n}$ for any positive integer $n$, and also for any negative integer $n$.

Let us take some $h \in G$. Then $\Delta\left(h a_{1}\right)=h \otimes h a_{1}+h a_{1} \otimes h g$ and $h a_{1} \notin C_{0}$, so $P_{h g, h}(C) \neq K(h g-h)$. Hence there is an arrow starting at $h$ and ending at $h g$ in $C$; as before, inductively we get that there are in $C$ arrows

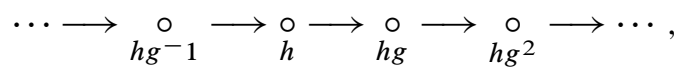

which shows that the vertex $h$ belongs to a connected component $D$ of type $K\left[\mathbb{A}_{\infty} \mid s^{\prime}\right]$ for some $s^{\prime} \geq 1$. Moreover, $\Delta\left(a_{1} h\right)=h \otimes a_{1} h+a_{1} h \otimes g h$, we also have $P_{g h, h}(C) \neq$ $K(g h-h)$, so there is an arrow from $h$ to $g h$ in $C$. This shows that $h g=g h$, so then $g$ must lie in $Z(G)$. then

If we denote by $p_{h, g^{i} h}$ the unique path from $h$ to $g^{i} h$, for any $h \in G$ and $i \geq 0$,

$$
\Delta\left(p_{1, g} s\right)-1 \otimes p_{1, g^{s}}-p_{1, g^{s}} \otimes g^{s} \in C_{s-1} \otimes C_{s-1}
$$

and $p_{1, g} \notin \notin C_{s-1}$. Then

$$
\Delta\left(h p_{1, g} s\right)-h \otimes h p_{1, g^{s}}-h p_{1, g^{s}} \otimes h g^{s} \in C_{s-1} \otimes C_{s-1}
$$

and $h p_{1, g} \ell_{s-1}$. But it is easy to check that in the path coalgebra $K \Gamma$ (whose subcoalgebra is $C$ ) the relation $\Delta(c)-h \otimes c-c \otimes h g^{s} \in(K \Gamma)_{s-1} \otimes(K \Gamma)_{s-1}$ holds if and only if $c \in(K \Gamma)_{s-1}+K p_{h, h g} s$. Applying this for $c=h p_{1, g} \notin \notin C_{s-1}$, we obtain $h p_{1, g} s=c^{\prime}+\gamma p_{h, h g}$ for some $c^{\prime} \in(K \Gamma)_{s-1}$ and $\gamma \in K^{*}$. This shows 
that $p_{h, h g} s$ must be in $C$, so it also lies in $D$, which implies that $s^{\prime} \geq s$ (otherwise $D$ cannot have paths of length $s$ ).

Similarly, since

$$
\Delta\left(h^{-1} p_{h, h g s^{\prime}}\right)-1 \otimes h^{-1} p_{h, h g^{s^{\prime}}}-h^{-1} p_{h, h s^{s^{\prime}}} \otimes g^{s^{\prime}} \in C_{s^{\prime}-1} \otimes C_{s^{\prime}-1}
$$

and $h^{-1} p_{h, h s^{s^{\prime}}} \notin C_{s^{\prime}-1}$, we obtain $s \geq s^{\prime}$. In conclusion $s^{\prime}=s$, and $C$ is a direct sum of coalgebras isomorphic to $K\left[\mathbb{A}_{\infty} \mid s\right]$. Moreover, this direct sum is indexed by a set in bijection with $G /\langle g\rangle$.

In order to uncover the Hopf algebra structures on $C$, we use the lifting method proposed in [2]. Since $C_{0}=K \Gamma$ is a Hopf subalgebra of $C$, the coradical filtration $C_{0} \subseteq C_{1} \subseteq \cdots$ of $C$ is a Hopf algebra filtration, and we can consider the associated graded space gr $C=C_{0} \oplus \frac{C_{1}}{C_{0}} \oplus \ldots$, which has a graded Hopf algebra structure. Denote $H=K \Gamma$, the degree 0 component of gr $C$, and by $\gamma: H \rightarrow \operatorname{gr} C$ the inclusion morphism. The natural projection $\pi: \operatorname{gr} C \rightarrow H$ is a Hopf algebra morphism. Then the coinvariants $R=(\operatorname{gr} C)^{c o H}$ with respect to the right $H$-coaction induced via $\pi$, i.e.,

$$
R=\{z \in \operatorname{gr} C \mid(I \otimes \pi) \Delta(z)=z \otimes 1\}
$$

is a left Yetter-Drinfeld module over $H$, with left $H$-action defined by $h \cdot r=$ $\sum \gamma\left(h_{1}\right) r S\left(\gamma\left(h_{2}\right)\right)$ for any $h \in H, r \in R$, and left $H$-coaction $\delta(r)=\sum r_{(-1)} \otimes$ $r_{(0)}=(\pi \otimes I) \Delta(r)$. Moreover, $R$ is a graded subalgebra of $\operatorname{gr} C$, with grading denoted by $R=\bigoplus_{n \geq 0} R(n)$, and it also has a coalgebra structure with comultiplication $\Delta_{R}(r)=\sum r^{(1)} \otimes r^{(2)}=\sum r_{1} \gamma \pi\left(S\left(r_{2}\right)\right) \otimes r_{3}$, and these make $R$ a braided Hopf algebra in the category ${ }_{H}^{H} Y D$ of Yetter-Drinfeld modules over $H$. The Hopf algebra gr $C$ can be reconstructed from $R$ by bosonization, i.e., gr $C \simeq R \# H$, the biproduct of $R$ and $H$. The multiplication of this biproduct is the smash product given by $(r \# h)(p \# v)=\sum r\left(h_{1} \cdot p\right) \# h_{2} v$, while the comultiplication is the smash coproduct $\Delta(r \# h)=\sum\left(r^{(1)} \#\left(r^{(2)}\right)_{(-1)} h_{1}\right) \otimes\left(r^{(2)}\right)_{(0)} \# h_{2}$.

Since in our case $C_{i}$ is the span of all paths of length at most $i$ in $C$, if $z=$ $\hat{c} \in R(n)$, then $c=\sum_{i} \alpha_{i} p_{i}$, a linear combination of paths $p_{i}$ of length $i$, and $\sum_{i} \alpha_{i} \widehat{p}_{i} \otimes t\left(p_{i}\right)=\sum_{i} \alpha_{i} \widehat{p}_{i} \otimes 1$. Then $\alpha_{i}=0$ for any $i$ such that $t\left(p_{i}\right) \neq 1$, showing that $R(i)$ is spanned by the classes of the paths of length $i$ which end at 1 . We conclude that $R(i)$ has dimension 1 for any $0 \leq i \leq s$, and $\operatorname{dim}(R)=s+1$. By [2], Theorem 3.2 (see also [9], Proposition 3.4) $R$ is isomorphic to a quantum line, i.e., $R \simeq R_{q}(H, v, \chi)$ for some primitive $(s+1)$-st root of unity $q$, an element $v \in G$ and a character $\chi \in G^{*}$ such that $\chi(v)=q$, and $\chi(h) h v=\chi(h) v h$ for any $h \in G$, i.e., $v \in Z(G)$ (we use the notation of [9], Section 2). As an algebra we have $R_{q}(H, v, \chi)=K[y] /\left(y^{s+1}\right)$, and the coalgebra structure is such that the elements $d_{0}=1, d_{1}=y, d_{2}=\frac{y^{2}}{(2)_{q} !}, \ldots, \frac{y^{s}}{(s)_{q} !}$ form a divided power sequence, i.e., $\Delta\left(d_{i}\right)=\sum_{0 \leq j \leq i} d_{j} \otimes d_{i-j}$ for any $0 \leq i \leq s$. The $H$-action on $R_{q}(H, v, \chi)$ is such that $h \cdot y=\chi(h) y$ for any $h \in G$, and the $H$-coaction is such that $y \mapsto v \otimes y$.

By [9], Proposition 3.1, there exists a $(1, v)$-skew-primitive $z$ in $C$, which is not in $C_{0}$, such that $v z=q z v, C$ is generated as an algebra by $z$ and $G$, and the class 
$\hat{z}$ in $\frac{C_{1}}{C_{0}}$ corresponds to the element $y \# 1$ in $R_{q}(H, v, \chi) \# H$ via the isomorphism gr $C \simeq R_{q}(H, v, \chi) \# H$. It follows that $v$ must be $g^{-1}$. Since for $h \in G$ both $z h$ and $h z$ are $\left(h, g^{-1} h\right)$-skew-primitives, we must have $z h=\lambda h z+\beta\left(g^{-1} h-h\right)$ for some scalars $\lambda$ and $\beta$. But $z h g=\left(\lambda h z+\beta\left(g^{-1} h-h\right)\right) g=q \lambda h g z+\beta(h-h g)$, and on the other hand $z g h=q g z h=q \lambda g h z+q \beta(h-h g)$, showing that $\beta=0$. Thus $z h=\lambda h z$, and passing to gr $C$, this gives $\hat{z} h=\lambda h \hat{z}$. But in $R_{q}(H, v, \chi) \# H$ we have $(1 \# h)(y \# 1)=\chi(h)(y \# 1)(1 \# h)$, so $\lambda=\chi(h)$. Therefore $z h=\chi(h) h z$. Replace the generator $z$ by $x=g z$, which is a $(g, 1)$-skew-primitive. By the quantum binomial formula we see that $\Delta\left(x^{s+1}\right)=1 \otimes x^{s+1}+x^{s+1} \otimes g^{s+1}$, so then $x^{s+1}=\alpha\left(g^{s+1}-1\right)$ for some scalar $\alpha$. Since $x^{s+1} h=\chi(h)^{s+1} h x^{s+1}$, we see that if $\chi^{s+1} \neq 1$, then $\alpha$ must be zero. Now it is clear that $C \simeq H_{\infty}\left(s, q^{-1}, G, g, \chi, \alpha\right)$.

Case 2. If 1 is a vertex in a connected component $D$ of type $K\left[\mathbb{C}_{1}, s\right]$, with $s \geq 1$, then let $x$ be the arrow from 1 to 1 , which is a primitive element, i.e., $\Delta(x)=x \otimes 1+1 \otimes x$. Then $g x \notin C_{0}$ and $\Delta(g x)=g x \otimes g+g \otimes g x$ for any $g \in G$, so there is an arrow from $g x$ to $g x$. This shows that $C$ must be a direct sum of coalgebras of type $K\left[\mathbb{C}_{1}, s^{\prime}\right]$ (for possible different values of $s^{\prime}$ ). Then looking at $\Delta\left(x^{i}\right)-x^{i} \otimes 1-1 \otimes x^{i}$, it is easy to show by induction that $x^{i}$ lies in $D$ for any $i \geq 1$. Since $x$ is a non-zero primitive element, the $\operatorname{set}\left(x^{i}\right)_{i \geq 1}$ is linearly independent, a contradiction to the finite dimensionality of $D$. Thus this situation cannot occur.

If 1 is a vertex in a connected component of type $K\left[\mathbb{C}_{n}, s\right]$, with $n \geq 2$, the proof goes as in case 1, and leads us to the conclusion that $C$ is a direct sum of coalgebras isomorphic to $K\left[\mathbb{C}_{n}, s\right]$, and that $C$ is isomorphic as a Hopf algebra to one of the form $H_{n}(s, q, G, g, \chi, \alpha)$. The only difference is that instead of using the paths $p_{h, g^{i} h}$, we deal with paths denoted by $p_{h \mid l}$, and meaning the path of length $l$ starting at the vertex $h$. Also, since $\chi(g)=q$, a $(s+1)$-st root of unity, and $g^{n}=1, s+1$ must divide $n$.

Case 3. If 1 is a vertex in a connected component of type $K$, then proceeding as in case 1 , we can see that there are no arrows in $C$, so $C$ is a direct sum of copies of $K$. Thus $C$ is a group-like coalgebra, and Hopf algebra structures on $C$ are just group Hopf algebras.

We note that the above theorem completely classifies finite dimensional Hopf algebras whose underlying algebras are quotients of finite dimensional path algebras by ideals generated by paths, or whose underlying coalgebras are path subcoalgebras. These are the algebras $K G, H_{n}(s, q, G, g, \chi, \alpha)$ and their duals, because a finite dimensional Hopf algebra is Frobenius as an algebra and co-Frobenius as a coalgebra.

Acknowledgment. The research of the first and the third authors was supported by Grant ID-1904, contract 479/13.01.2009 of CNCSIS. For the second author, this work was supported by the strategic grant POSDRU/89/1.5/S/58852, Project "Postdoctoral programe for training scientific researchers" cofinanced by the European Social Fund within the Sectorial Operational Program Human Resources Development 2007-2013. 


\section{References}

[1] M. Aguiar and W. Ferrer Santos, Galois connections for incidence Hopf algebras of partially ordered sets. Adv. Math. 151 (2000), 71-100. Zbl 0945.06002 MR 1752242

[2] N. Andruskiewitsch and H.-J. Schneider, Lifting of quantum linear spaces and pointed Hopf algebras of order $p^{3}$.J. Algebra 209 (1998), 658-691.Zbl 0919.16027 MR 1659895

[3] M. Artin and W. F. Schelter, Graded algebras of global dimension 3. Adv. in Math. 66 (1987), 171-216. Zbl 0633.16001 MR 917738

[4] M. Beattie, S. Dăscălescu, and L. Grünenfelder, Constructing pointed Hopf algebras by Ore extensions. J. Algebra 225 (2000), 743-770. Zbl 0948.16026 MR 1741560

[5] M. Beattie and R. Rose, Balanced bilinear forms on matrix and matrix-like coalgebras. Comm. Algebra 36 (2008), 1311-1319. Zbl 1147.16023 MR 2406586

[6] R. Berger, Koszulity for nonquadratic algebras. J. Algebra 239 (2001), 705-734. Zbl 1035.16023 MR 1832913

[7] R. Berger, Non-homogeneous $N$-Koszul algebras. Rev. Un. Mat. Argentina 48 (2007), 53-56 (2008). Zbl 1158.16305 MR 2389458

[8] J. Bichon, $N$-complexes et algèbres de Hopf. C. R. Math. Acad. Sci. Paris 337 (2003), 441-444. Zbl 1031.16020 MR 2023750

[9] C. Călinescu, S. Dăscălescu, A. Masuoka, and C. Menini, Quantum lines over non-cocommutative cosemisimple Hopf algebras. J. Algebra 273 (2004), 753-779. Zbl 1042.16022 MR 2037722

[10] X.-W. Chen, H.-L. Huang, Y. Ye, and P. Zhang, Monomial Hopf algebras. J. Algebra 275 (2004), 212-232. Zbl 1071.16030 MR 2047446

[11] W. Chin, M. Kleiner, and D. Quinn, Almost split sequences for comodules. J. Algebra 249 (2002), 1-19. Zbl 1005.16034 MR 1887982

[12] W. Chin and S. Montgomery, Basic coalgebras. In Modular interfaces (Riverside, CA, 1995), AMS/IP Stud. Adv. Math. 4, Amer. Math. Soc., Providence, RI, 1997, 41-47. Zbl 0920.16018 MR 1483902

[13] C. Cibils and M. Rosso, Hopf quivers. J. Algebra 254 (2002), 241-251. Zbl 1020.16025 MR 1933868

[14] A. Connes and M. Dubois-Violette, Yang-Mills algebra. Lett. Math. Phys. 61 (2002), 149-158. Zbl 1028.53025 MR 1936574

[15] S. Dăscălescu, C. Năstăsescu, and Ş. Raianu, Hopf algebras. Monographs Textbooks Pure Appl. Math. 235, Marcel Dekker, New York 2001. Zbl 0962.16026 MR 1786197

[16] S. Dăscălescu, C. Năstăsescu, and G. Velicu, Balanced bilinear forms and finiteness properties for incidence coalgebras over a field. Rev. Un. Mat. Argentina 51 (2010), 19-26. Zbl 1218.16024 MR 2681257

[17] E. L. Green, E. N. Marcos, R. Martínez-Villa, and P. Zhang, D-Koszul algebras. J. Pure Appl. Algebra 193 (2004), 141-162. Zbl 1075.16013 MR 2076383

[18] E. L. Green and $\varnothing$. Solberg, Basic Hopf algebras and quantum groups. Math. Z. 229 (1998), 45-76. Zbl 0924.16028 MR 1649318 
[19] M. C. Iovanov, Co-Frobenius coalgebras. J. Algebra 303 (2006), 146-153. Zbl 1104.16030 MR 2253657

[20] M. C. Iovanov, Abstract integrals in algebra: coalgebras, Hopf algebras and compact groups. Internat. J. Math., to appear; preprint 2008. arXiv:0810.3740

[21] M. C. Iovanov, Generalized Frobenius algebras and the theory of Hopf algebras; Canad. J. Math., Doi 10.4153/CJM-2012-060-7, to appear; preprint 2008. arXiv:0803.0775

[22] S. A. Joni and G.-C. Rota, Coalgebras and bialgebras in combinatorics. Stud. Appl. Math. 61 (1979), 93-139. Zbl 0471.05020 MR 544721

[23] S. Montgomery, Hopf algebras and their actions on rings. CBMS Regional Conf. Ser. in Math. 82, Amer. Math. Soc., Providence, RI, 1993. Zbl 0793.16029 MR 1243637

[24] W. D. Nichols, Bialgebras of type one. Comm. Algebra 6 (1978), 1521-1552. Zbl 0408.16007 MR 0506406

[25] W. R. Schmitt, Incidence Hopf algebras. J. Pure Appl. Algebra 96 (1994), 299-330. Zbl 0808.05101 MR 1303288

[26] D. Simson, Incidence coalgebras of intervally finite posets, their integral quadratic forms and comodule categories. Colloq. Math. 115 (2009), 259-295. Zbl 1173.16009 MR 2491748

Received July 14, 2011

S. Dăscălescu, M. C. Iovanov, C. Năstăsescu, University of Bucharest, Facultatea de Matematica si Informatica, Str. Academiei 14, Bucharest 1, 010014, Romania

E-mail: sdascal@fmi.unibuc.ro; Constantin_nastasescu@yahoo.com

M. C. Iovanov, University of Southern California, 3620 S Vermont Ave, KAP 108, Los Angeles, CA 90089, U.S.A.

E-mail: yovanov@gmail.com 